\title{
Adaptación y Validación de la Escala de Orientación a la Dominancia Social 7 en Colombia
}

\section{Adaptation and Validation of the Social Dominance Orientation Scale 7 in Colombia}

\author{
Efraín García-Sánchez ${ }^{1,2}$, Nelson Molina-Valencia ${ }^{3}$, Estefanía Buitrago Sánchez $^{3}$, \\ Valentina Ramírez González ${ }^{3}$, Zabdy Sanz ${ }^{3}$ y Andrés Tello Cifuentes ${ }^{3}$ \\ ${ }^{1}$ Departamento de Psicología Social, Universidad de Granada \\ 2 Núcleo de Estudios de la Violencia, Universidade de São Paulo \\ ${ }^{3}$ Facultad de Psicología, Universidad del Valle
}

\begin{abstract}
La orientación a la dominancia social (SDO) se define como la disposición individual a aceptar las relaciones jerárquicas y de dominación entre grupos. La medición de la SDO ha evolucionado conforme con el desarrollo de la teoría, aportándose varias versiones de la escala. Esta escala ha mostrado indicadores de confiabilidad y validez apropiados en diferentes contextos, pero también presenta algunas limitaciones de método relacionadas con la redacción de los ítems y potenciales ambigüedades de contenido. Se presenta la adaptación y validación de la escala $\mathrm{SDO}_{7}$ en español para el contexto colombiano, en la cual se subsanan algunas limitaciones de la escala original. En 2 estudios realizados en una muestra intencional de población universitaria de Cali, Colombia ( $\left.n_{\text {Estudio1 }}=394, n_{\text {Estudio2 }}=867\right)$ se aporta evidencia sobre las propiedades psicométricas de la escala y la validez predictiva de sus dimensiones sobre actitudes sociales/políticas en el contexto colombiano. Los análisis factoriales ratifican la estructura factorial de 2 dimensiones de SDO (anti-igualitarismo y dominancia). Además, los análisis de regresión sugieren que las dimensiones de dominancia y anti-igualitarismo se asocian de forma diferenciada con actitudes políticas que justifican la desigualdad: la dominancia se asoció con medidas explícitas y hostiles, mientras que el anti-igualitarismo lo hizo más con medidas sutiles que mantienen las desigualdades. Este estudio contribuye a la comprensión de uno de los procesos asociados con la legitimación de la desigualdad y al desarrollo de herramientas para su investigación empírica.
\end{abstract}

Palabras clave: orientación a la dominancia social, desigualdad, ideologías, actitudes políticas, Colombia

Social dominance orientation (SDO) is defined as the individual disposition to accept hierarchy and dominance among groups. The measurement of SDO has evolved accordingly with the development of the theory, providing several versions of this scale. This scale has demonstrated indicators of reliability and validity across contexts, but it also has some limitations related to potential ambiguities and the phrasing of items. In this article we present the adaptation and validation of the $\mathrm{SDO}_{7}$ scale in Spanish for the Colombian context, where some of these limitations are addressed. In 2 studies conducted with conventional samples $\left(n_{\text {Study } 1}=394 ; n_{\text {Study } 2}=867\right)$, we provide evidence for the psychometric properties of the scale and the predictive validity of its dimensions on social/political attitudes within the Colombian context. Factor analyses confirmed the factorial structure of two substantive dimensions of SDO (dominance and antiegalitarianism). Regression analyses suggested that both dimensions, dominance and anti-egalitarianism, were accordingly associated with different attitudes that justify inequality: Dominance was associated with more explicit and hostile attitudes, whereas anti-egalitarianism was linked to subtler measures that maintain inequality. This article contributes to the comprehension of one of the processes related to the legitimacy of inequality and to the development of tools for its empirical research.

Keywords: social dominance orientation, inequality, ideology, political attitudes, Colombia

Efraín García-Sánchez

https://orcid.org/0000-0002-8097-5822

Nelson Molina-Valencia iD https://orcid.org/0000-0002-0250-251X

Este proyecto fue realizado gracias a la beca de formación doctoral otorgada al primer autor por el Departamento Administrativo de Ciencia, Tecnología e Innovación (COLCIENCIAS) $\mathrm{N}^{\circ} 679$. El material suplementario, datos y archivos utilizados en esta investigación se encuentran disponibles en https://osf.io/nxmwp/. Todos/as los/as autores/as contribuyeron sustantivamente en este artículo: el primer y segundo autor diseñaron el estudio; el primer, tercer, cuarto, quinto y sexto autores/as recolectaron la información y revisaron los materiales del manuscrito; el primer autor realizó los análisis y escribió el primer borrador del manuscrito. Todos/as los/as autores/as realizaron contribuciones en la revisión y lectura y han aprobado el manuscrito final. No existe ningún conflicto de intereses que revelar.

La correspondencia relativa a este artículo debe ser dirigida a Efraín García-Sánchez, Departamento de Psicología Social, Centro de Investigación en Mente, Cerebro y Comportamiento, Universidad de Granada, Campus de Cartuja, S/N, C.P. 18012, Granada, España. Email: egarcias@ugr.es 
Los conflictos intergrupales han estado caracterizados por las desigualdades sociales. Estas desigualdades responden a dinámicas estructurales, políticas y económicas, así como a algunas características psicológicas. La teoría de la dominancia social (TDS) plantea que las personas tienen una orientación a favorecer la dominación de unos grupos sobre otros y aceptar las jerarquías sociales, lo que (re)produce las desigualdades sociales (Pratto et al., 1994; Sidanius \& Pratto, 1999). Este constructo, denominado orientación a la dominancia social (SDO por sus siglas en inglés, social dominance orientation; Pratto et al., 1994), se refuerza en contextos más desiguales y con mayor prevalencia de conflictos intergrupales (Kunst et al., 2017; Pratto et al., 2013).

El uso de la escala de SDO se ha extendido en estudios transculturales, mostrando una consistencia interna y validez predictiva adecuada en diferentes contextos (Pratto et al., 2013; Vargas-Salfate et al., 2018). Existen diferentes versiones de la escala de SDO en castellano en Chile (Cárdenas et al., 2010), Argentina (Jaume et al., 2012) y España (Silván Ferrero \& Bustillos, 2007). Sin embargo, estas adaptaciones están basadas en versiones iniciales de la SDO, que presentan al menos dos limitaciones. Primero, la redacción de los ítems en un mismo sentido (pro-rasgo) que no permite controlar por la varianza derivada de la redacción. Segundo, el contenido de algunos ítems se podría solapar con otros constructos y procesos intergrupales. Por tal motivo, Ho et al. (2015) realizaron una nueva versión de la escala de Orientación a la Dominancia Social $\left(\mathrm{SDO}_{7}\right)$, en la que se corrigen algunas de estas limitaciones.

El objetivo de nuestro estudio fue realizar la adaptación y validación de la escala de $\mathrm{SDO}_{7}$ en el contexto colombiano. Para esto presentamos evidencia empírica de la confiabilidad y validez predictiva de la $\mathrm{SDO}_{7}$ sobre actitudes sociales y políticas. De este modo, contribuimos a la investigación psicológica sobre legitimación de la desigualdad de distintas formas. Primero, proporcionamos una versión mejorada de la SDO en castellano; segundo, aportamos información sobre la validez predictiva de cada una de sus dimensiones y tercero, presentamos un instrumento adaptado al contexto colombiano que facilite la investigación empírica sobre la legitimación de las desigualdades sociales.

\section{Orientación a la Dominancia Social}

La SDO se define como una disposición psicológica mediante la cual las personas avalan la dominación y la desigualdad entre grupos (Pratto et al., 1994). Aunque a veces se refiere a la SDO como una ideología en sí misma, algunos autores argumentan que la SDO no se reduce a una serie de creencias específicas sobre el mundo o sobre ciertos grupos en particular (e.g., minorías étnicas, pobres, inmigrantes), sino que se trata de una disposición individual que exacerba la adherencia a ciertas ideologías —denominadas mitos legitimadores - que justifican las relaciones de dominación y desigualdad (Pratto et al., 2013; Sibley \& Duckitt, 2010). Esto significa que las ideologías son indicadores del grado de SDO de las personas. De hecho, la SDO se relaciona con ideologías que justifican la desigualdad y promueven la dominación intergrupal, tales como el conservadurismo político, las creencias en el mundo justo, el patriotismo, el sexismo y la ética protestante, entre otras (Cotterill et al., 2014; Pratto et al., 1994; Sidanius et al., 2017). Sin embargo, la SDO no se reduce a tales ideologías, sino que estas intervienen como mediadoras en la relación que hay entre la SDO y las actitudes políticas (Grina et al., 2016; Sibley \& Duckitt, 2010).

La SDO varía en función de algunas diferencias individuales. Por ejemplo, en diferentes contextos se ha encontrado que los hombres están más orientados a la dominación social que las mujeres y promueve una mayor adhesión a actitudes sociales conservadoras (Cárdenas et al., 2010; Etchezahar et al., 2014; Jost \& Thompson, 2000; Pratto et al., 1994; Sidanius \& Pratto, 1999). También se ha encontrado que la SDO se asocia positivamente con el nacionalismo (Cárdenas et al., 2010; Pratto et al., 1994), el apoyo a políticas que mantienen la dominación y la inequidad (Cárdenas et al., 2010; Duckitt \& Sibley, 2007), la justificación del sistema económico (Etchezahar et al., 2014), la legitimidad de las instituciones (Janos et al., 2018), la configuración política conservadora (Carvacho et al., 2013), el rechazo a la multiculturalidad (Sirlopú et al., 2015) y el autoritarismo (Altemeyer, 1998; Duckitt, 2006; Silván Ferrero \& Bustillos, 2007; Etchezahar et al., 2014).

Aunque originalmente la SDO se planteó como un constructo unidimensional (Pratto et al., 1994), empíricamente se ha demostrado la existencia de dos dimensiones: Dominancia Intergrupal (SDO-D) y Antiigualitarismo (SDO-A, también llamada oposición a la igualdad; Cárdenas et al., 2010; Etchezahar et al., 2014; Jost \& Thompson, 2000; Silván Ferrero \& Bustillos, 2007). La SDO-D se refiere a la opresión de unos grupos con poder - material o simbólico - sobre otros grupos, de manera que se mantiene una relación de subordinación explícita. El SDO-A, por su parte, hace referencia a la preferencia por las relaciones jerárquicas que perpetúan las desigualdades grupales. 
Estas dos dimensiones hacen parte del mismo constructo de SDO, pero tienen características diferentes en cuanto a su relación con comportamientos y actitudes sobre la desigualdad. La SDO-D se encuentra positivamente asociada con la deshumanización (Kteily et al., 2015), el apoyo a la tortura (Larsson et al., 2012), el racismo, el nacionalismo, el militarismo, la guerra y políticas que buscan castigar a los otros grupos (Ho et al., 2015). El SDO-A, por su parte, se asocia con mayor rechazo a los inmigrantes (Martinovic \& Verkuyten, 2013), menor apoyo al multiculturalismo (Hindriks et al., 2014), menor apoyo a la redistribución de recursos, menor apoyo a formas de gobierno democráticas (Ellenbroek et al., 2014), mayor conservadurismo político, oposición a acciones afirmativas, justificación del sistema y racismo simbólico (Ho et al., 2015). Así, mientras que la SDO-D refleja formas de legitimación de la desigualdad de forma explícita y hostil, el SDO-A refleja formas de legitimación de la desigualdad más sutil, basadas en el apoyo a medidas sociales.

\section{Escalas de Medición de la SDO}

Inicialmente la escala SDO estaba compuesta por 14 ítems que se organizaban en una dimensión ( $\alpha$ de Cronbach = 0,83); posteriormente Pratto et al. (1994) elaboraron una versión de 16 ítems $(\alpha=0,91)$, que es la versión más utilizada actualmente. Esta escala, tanto en su versión completa como en algunas versiones reducidas, ha demostrado tener un poder predictivo adecuado para dar cuenta de actitudes y comportamientos intergrupales a través del tiempo y en diferentes contextos culturales (Kunst et al., 2017; Pratto et al., 2013; Vargas-Salfate et al., 2018). Por tal motivo, la SDO se ha constituido como un constructo sólido dentro de la psicología social y política para investigar actitudes hacia la desigualdad y conflictos intergrupales (Ho et al., 2015).

Actualmente existen tres versiones de la escala SDO en castellano: una adaptación en España (16 ítems, $\alpha=0,85$; Silván Ferrero \& Bustillos, 2007), una en Chile (16 ítems, a = 0,86; Cárdenas et al., 2010) y una en Argentina (10 ítems, $\alpha=0,85$; Etchezahar et al., 2014). Estas versiones están basadas en la versión original de 16 ítems $\left(\mathrm{SDO}_{6}\right)$ propuesta por Pratto et al. (1994), la cual ha presentado niveles de confiabilidad y validez adecuados. Pero esta escala también tiene algunas limitaciones identificadas por Ho et al. (2015). Primero, la redacción de los ítems de la dimensión SDO-A está en positivo (pro-trait), es decir, no hay ítems inversos que permitan controlar fuentes secundarias de varianza derivadas del método (Cronbach, 1995).

Segundo, las versiones en castellano muestran asociaciones con otras variables ideológicas (e.g., justificación del sistema económico, ideología política), pero no se ha aportado evidencia sobre la validez predictiva de cada una de las dimensiones de la SDO sobre diferentes actitudes políticas. Tercero, la redacción de los ítems cambia entre versiones, bien sea por una cuestión de adaptación cultural o bien por el contenido de los ítems, lo cual podría afectar la validez de constructo. Por ejemplo, el ítem "A veces es necesario utilizar la fuerza contra otros grupos para conseguir lo que $t u$ grupo quiere" (Silván Ferrero \& Bustillos, 2007) podría hacer referencia a un sesgo endogrupal (teoría de la identidad social) o el ítem "Para progresar en la vida, en ocasiones es necesario pasar por encima de otras personas" (Cárdenas et al., 2010) se refiere a una creencia prescriptiva general, que no necesariamente refleja dinámicas intergrupales. Estas limitaciones metodológicas son propias de la versión original del inglés y se subsanan en la versión 7 de la escala $\left(\mathrm{SDO}_{7}\right)$ propuesta por Ho et al. (2015). En esta versión se realizó una revisión de todos los ítems, se incluyeron algunas variaciones y se tuvo especial cuidado en contrabalancear el sentido de los ítems (a favor y en contra) y mantener siempre la dimensión intergrupal.

\section{La Presente Investigación}

El objetivo de nuestra investigación fue adaptar y validar la escala $\mathrm{SDO}_{7}$ en el contexto colombiano. Siguiendo las recomendaciones dadas por la Comisión Internacional de Tests (Muñiz et al., 2013), en primer lugar, buscamos replicar la estructura factorial de la $\mathrm{SDO}_{7}$, controlando las limitaciones de método de la escala, siguiendo el mismo procedimiento realizado por Ho et al. (2015). Además, aportamos evidencia de la validez predictiva de las dimensiones de la $\mathrm{SDO}_{7}$ sobre algunas ideologías y actitudes políticas asociadas a la legitimación de la desigualdad.

Nuestra primera hipótesis de trabajo $\left(\mathrm{H}_{1}\right)$ fue que la escala presenta un ajuste apropiado para una estructura de dos dimensiones conceptuales, controlando por los dos factores de método subyacentes a la redacción de las preguntas. De acuerdo con la TDS, las personas que hacen parte de grupos sociales privilegiados tenderían a reportar mayores niveles de SDO (Pratto et al., 1994; Sidanius et al., 2000; Sidanius $\&$ Pratto, 1999). Por tanto, en la segunda y tercera hipótesis de trabajo planteamos que los hombres reportan 
mayores niveles de SDO que las mujeres $\left(\mathrm{H}_{2}\right)$ y que el estatus socioeconómico se asocia positivamente con la SDO $\left(\mathrm{H}_{3}\right)$. En cuanto a la validez predictiva de las dimensiones, la dimensión SDO-D tiene una relación positiva con indicadores asociados al sometimiento y subordinación explícita de unos grupos sobre otros, tales como el apoyo a la solución militar del conflicto, el castigo físico a los niños y el patriotismo y adherirse más a valores como la autoridad, la influencia, la disciplina y la riqueza $\left(\mathrm{H}_{4 a}\right)$. Por su parte, la dimensión SDO-A está asociada positivamente con medidas más sutiles de legitimación de la desigualdad, tales como la justificación del sistema económico, el rechazo a la protesta social, la justificación de la desigualdad, el rechazo a la inversión social, la inclusión educativa, las actitudes hacia la redistribución de recursos y una menor aceptación de valores como la paz, la justicia y la igualdad $\left(\mathrm{H}_{4 b}\right)$.

En dos estudios independientes, verificamos las propiedades psicométricas de la escala y aportamos evidencias de su validez predictiva sobre variables ideológicas y actitudinales. En el Estudio 1, incluimos una serie más amplia de indicadores actitudinales y de valores sociales. En el Estudio 2, la muestra fue más grande y diversa, con lo cual pudimos evaluar la invarianza del instrumento por sexo y ver diferencias según las variables sociodemográficas. Debido a que ambos estudios siguen el mismo procedimiento, los presentamos simultáneamente en el Método y los Resultados.

\section{Método}

\section{Participantes}

El Estudio 1 estuvo conformado por 394 estudiantes universitarios seleccionados de forma intencional $\left(M_{\text {edad }}=20,33, D T=3,59 ; 52 \%\right.$ mujeres $)$ en las facultades de ciencias, salud, ingeniería, psicología, trabajo social, artes integradas, educación y pedagogía de cinco universidades de la ciudad de Cali, Colombia $(84,19 \%$ participantes de universidad pública y $15,81 \%$ de universidad privada). Las personas aceptaron participar voluntariamente en un estudio sobre temas sociales de actualidad en Colombia. La muestra del Estudio 2 estuvo compuesta por 867 personas de la comunidad académica de una universidad pública en Cali, Colombia (estudiantes, trabajadores/as y profesores/as), $M_{\text {edad }}=29,77, D T=12,74 ; 55 \%$ mujeres). Los/as participantes fueron invitados/as a hacer parte del estudio a través de la lista de distribución de correos de la universidad. Las personas interesadas accedieron voluntariamente y dieron su consentimiento para participar en el estudio (1,9\% reportó formación de secundaria, $5,3 \%$, educación técnica o tecnológica, $65,3 \%$, estudiantes de grado, 4,4\%, título de grado de especialización, $14 \%$, de maestría y 9,1\% de doctorado).

\section{Medidas}

\section{Orientación a la Dominancia Social 7 (SDO 7$)$}

Usamos una versión traducida y adaptada de la $\mathrm{SDO}_{7}$ (Ho et al., 2015). Esta escala tiene 16 ítems (8 de cada dimensión), sobre los cuales los/as participantes debían indicar qué tan a favor o en contra se encontraban con cada uno de ellos en una escala que iba de 1 (Completamente en contra) a 7 (Completamente a favor), de modo que valores más altos indican una mayor orientación a la dominancia social (e.g. de ítem de SDO-D, "Algunos grupos de personas deberían ser mantenidos en su posición social"; e.g. de ítem de SDOA, "No deberíamos promover la igualdad entre grupos de personas"; ver la Tabla A del Anexo). La validez de constructo se encuentra respaldada por el amplio desarrollo conceptual de la TDS y las aplicaciones de la $\mathrm{SDO}_{7}$ en la literatura a nivel transcultural, cuya fiabilidad suele ser consistente (e.g., aCronbach $>0,70$, Ho et al., 2015; Pratto et al., 2013). En todas las medidas que describimos a continuación usamos esta misma escala de medida, exceptuando las preguntas sobre valores sociales y las variables sociodemográficas.

\section{Justificación del Sistema Económico}

Consiste en la naturalización y aceptación del modelo económico neoliberal. En el Estudio 1 seleccionamos aleatoriamente cinco ítems de la versión de siete ítems adaptada al castellano (Jaume et al., 2012) (e.g., "Si la gente trabaja duro, casi siempre consigue lo que quiere"). En el Estudio 2 seleccionamos solo tres ítems de esta escala para reducir el tiempo de respuesta del cuestionario $\left(M_{\text {Estudio1 }}=4,31, D T=1,34\right.$, $\alpha_{\text {Cronbach }}=0,79$; $M_{\text {Estudio2 }}=4,08, D T=1,39$, a Cronbach $=0,51$ ). Ver la Tabla B del Anexo. Calculamos la variable a través del promedio de los ítems, de modo que valores más altos indican una mayor justificación del sistema. 


\section{Patriotismo}

Se refiere al grado de identificación y apoyo con el país. La calculamos como la media de dos ítems adaptados libremente de encuestas sociales (i.e., "Siento un gran orgullo por nuestro país Colombia"; "Aunque a veces no esté de acuerdo con el gobierno, mi compromiso con Colombia siempre es muy fuerte"). Mayores valores de esta variable, indica un mayor patriotismo $\left(M=5,34, D T=1,33, r_{\text {Pearson }}=0,37\right)$.

\section{Apoyo a Medidas Sociales y Politicas}

Dada la ausencia de escalas validadas en el contexto colombiano sobre temas ideológicos y políticos, decidimos incluir 11 indicadores diferentes comúnmente usados en encuestas sociales para medir actitudes políticas. En el Estudio 1 usamos 10 indicadores, mientras que en el Estudio 2 usamos 5. La reducción de ítems en el Estudio 2 se debió a la necesidad de reducir la extensión del cuestionario. Las respuestas estaban codificadas de modo que mayores valores de los ítems indicaban una mayor aceptación o apoyo a las medidas sociales o políticas mencionadas. Los indicadores que usamos hacían referencia al nivel de apoyo o aceptación de medidas sociales/políticas, como el matrimonio homosexual $\left(M_{\text {Estudio } 1}=4,43, D T=2,02\right)$, la pena de muerte ( $\left.M_{\text {Estudio }}=4,99, D T=2,17\right)$, el endurecimiento de penas $\left(M_{\text {Estudio } 2}=8,81, D T=1,66\right)$, el castigo físico a los niños/as por parte de sus padres $\left(M_{\text {Estudio } 1}=3,82, D T=1,92\right)$, la protesta social $\left(M_{\text {Estudio1 }}=5,93, D T=1,36\right.$; $\left.M_{\text {Estudio2 }}=5,37, D T=1,64\right)$, la inversión social $\left(M_{\text {Estudio1 }}=5,32, D T=1,69\right)$, la solución armada al conflicto $\left(M_{\text {Estudio } 1}=2,65, D T=1,76 ; M_{\text {Estudio } 2}=2,00, D T=1,50\right)$, la preocupación por la desigualdad $\left(M_{\text {Estudio1 }}=6,08\right.$, $\left.D T=1,36 ; M_{\text {Estudio } 2}=6,50, D T=1,08\right)$, la justificación de la desigualdad $\left(M_{\text {Estudio1 }}=3,62, D T=1,88\right)$ y la inclusión educativa $\left(M_{E s t u d i o 1}=5.55, D T=1.58\right)$. La redacción exacta de estos ítems se encuentra disponible en la Tabla B del Anexo. La redistribución de ingresos por parte del gobierno fue medida con un solo ítem en el Estudio 1 y con el promedio de 7 ítems adaptados de una escala de actitudes hacia la redistribución en el Estudio $2\left(M_{\text {Estudio } 1}=4,83, D T=1,73 ; M_{\text {Estudio } 2}=5,33, D T=0,92\right.$, aCronbach $\left.=0,68\right)$ (e.g., "El gobierno debería imponer mayores impuestos a las personas con más ingresos económicos") (García-Sánchez et al., 2021).

\section{Valores Sociales}

En consonancia con los estudios internacionales, incluimos ocho valores en el Estudio 1. Las personas debían indicar el grado de importancia de cada uno de ellos como principios que guían sus vidas. Para ello debían usar una escala entre 0 (Nada importante) a 7 (De suprema importancia); si estaban en contra de sus valores, podían señalar la opción -1. Los valores que preguntamos fueron: autodisciplina $(M=5,15, D T=1,74)$, honrar a los padres y mayores $(M=5,55, D T=1,64)$, igualdad $(M=5,77, D T=1,55)$, un mundo en paz $(M=5,96$, $D T=1,45)$, la justicia social $(M=6,09, D T=1,29)$, la autoridad $(M=4,28, D T=1,89)$, la influencia $(M=4,34$, $D T=1,96)$ y la riqueza $(M=3,50, D T=1,82)$ (ver la Tabla B del Anexo).

\section{Ideología Política}

En los dos estudios, se midió el autoposicionamiento político de las personas usando una escala que iba desde 1 (Extremadamente de izquierda) hasta 7 (Extremadamente de derecha) $\left(M_{\text {Estudio } 1}=3,55, D T=1,02\right.$; $\left.M_{\text {Estudio } 2}=3,23, D T=1,18\right)$.

\section{Estatus Socioeconómico}

El estatus socioeconómico objetivo lo medimos a través de los ingresos económicos y el nivel educativo. En los dos estudios las personas debían indicar en una escala ascendente de 1 a 10 la cantidad de ingresos económicos (en salarios mínimos) que reciben en su núcleo familiar $\left(M_{\text {Estudio1 }}=3,35, D T=2,20 ; M_{\text {Estudio2 }}=4,22\right.$, $D T=2,69)$. El nivel educativo se usó solo en el Estudio 2, ya que el Estudio 1 incluyó a estudiantes universitarios exclusivamente. Para medir el nivel educativo las personas debían señalar en una escala de 1 a 7, su último nivel educativo alcanzado o que estén adelantando (primaria, bachillerato, formación técnica, profesional universitario, especialización, maestría, doctorado) $\left(M_{\text {Estudio } 2}=4,50, D T=1,14\right)$. Adicionalmente, en ambos estudios incluimos la escala de estatus social subjetivo, en la cual los participantes debían indicar su posición social en una escalera que representa la sociedad colombiana. En las instrucciones se indicaba que en lo más alto de la escalera (10) estarían los grupos que tienen más dinero, mejores niveles educativos y los trabajos de más prestigio, mientras que en la parte más baja (1) estarían los grupos que tienen menos dinero, 
menores niveles educativos y trabajos de menor prestigio o están en desempleo $\left(M_{\text {Estudio } 1}=4,11, D T=1,52\right.$; $\left.M_{\text {Estudio2 }}=4,80, D T=1,69\right)$.

\section{Procedimiento}

La recolección de información la realizamos entre los meses de enero y mayo de 2018. El Estudio 1 fue realizado forma presencial por un equipo de investigadores entrenados para este fin y usando cuestionarios en papel. El Estudio 2 lo hicimos de forma virtual, usando la plataforma online Qualtrics. En ambos casos, las personas firmaron un consentimiento informado en el que indicábamos las condiciones del estudio, cumpliendo los criterios éticos estipulados por la Universidad de Granada. Estos estudios fueron aprobados por el comité de ética de la universidad coordinadora del proyecto sobre legitimación de la desigualdad social (No.170/CEIH/2016, Universidad de Granada). El tiempo de duración de respuesta del cuestionario fue entre 10 y 20 minutos aproximadamente.

\section{Plan de Análisis}

Este ejercicio de validación lo realizamos siguiendo las recomendaciones dadas por la Comisión Internacional de Tests (Muñiz et al., 2013). Hicimos una traducción directa del inglés al castellano, realizada por personas bilingües y con dominio tanto del contexto cultural como de investigación académica en psicología. Posteriormente, se realizó la traducción inversa del español al inglés por otras dos personas expertas. Nuestro equipo de investigación revisó las dos versiones y realizamos las adaptaciones pertinentes para la versión definitiva de la escala. Esta versión final fue puesta a prueba en un grupo de 20 personas (50\% hombres y $50 \%$ mujeres, con edades entre 18 y 60 años) para identificar posibles ajustes a los ítems, antes de realizar la recolección de la información. Los ítems originales y la versión al castellano se encuentran detallados en la Tabla A del Anexo.

El análisis de los datos siguió el protocolo utilizado por Ho et al. (2015). Así, realizamos un análisis descriptivo de los ítems para conocer las tendencias de las respuestas (media, desviación típica, asimetría, curtosis y correlación lineal de Pearson entre los ítems). También ejecutamos un análisis de confiabilidad

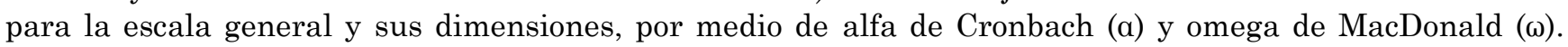
Estimamos la relación ítem-test usando el índice de correlación de Pearson y realizamos pruebas $t$ de Student para comparar las medias de las dimensiones de SDO (estimando el tamaño del efecto con la $d$ de Cohen). Hicimos análisis factoriales confirmatorios (AFC) para corroborar la estructura factorial de la escala. Para los AFC, utilizamos el estimador robusto de máxima verosimilitud, el cual es adecuado para escalas de tipo Likert con más de cinco opciones de respuesta y corrige algunas violaciones de la normalidad multivariada de los ítems (Brown, 2015). Seleccionamos los modelos tomando como referencia algunos de los criterios utilizados en la literatura: la raíz del error cuadrático medio de aproximación (RMSEA < 0,08, la raíz del residuo cuadrático medio estandarizado (SRMR $<0,06)$, el índice comparativo de Bentler-Bonett $(\mathrm{CFI}>0,90)$ y el índice de Tucker Lewis (TLI > 0,90) (Brown, 2015; Kline, 2016). En los dos estudios utilizamos los datos completos para mantener la mayor cantidad de información posible, aunque en los análisis eliminamos los casos en los que había valores perdidos (listwise deletion).

Para estimar los modelos, primero realizamos un AFC para una sola dimensión, luego un modelo de dos factores relativos al método (ítems en positivo y en negativo), luego de dos factores sustantivos (SDO-D y SDO-A) y, finalmente, una estructura de cuatro factores (dos conceptuales y dos de método; ver Figura 1). Finalmente, inspeccionamos los parámetros estimados del último modelo, con el fin de adaptarlo según algunas fuentes de desajuste focalizado que identificamos en el contexto colombiano. No encontramos violaciones de los supuestos estadísticos de los modelos (i.e., normalidad multivariante y errores residuales menores de 2).

Además, desarrollamos un análisis de invarianza para determinar si la estructura factorial se mantiene para distintos grupos según su sexo: invarianza configural (organización similar de los ítems por factor), métrica (equivalencia de las cargas factoriales), escalar (equivalencia de los interceptos de los ítems) y estricta (equivalencia de los residuos; Brown, 2015). El análisis de invarianza lo realizamos solo para el Estudio 2, debido a que cuenta con un tamaño de muestra adecuado para cada grupo $\left(n_{\text {Hombres }}=332, n_{\text {Mujeres }}=390\right)$. También estimamos un modelo de múltiples causas-múltiples indicadores (MIMIC), en el cual examinamos la influencia del sexo y el estatus socioeconómico sobre las dimensiones de SDO. Finalmente, realizamos análisis de regresión con las variables latentes para determinar el efecto de las dimensiones de $\mathrm{SDO}_{7}$ y diferentes actitudes, valores e ideologías. En estos análisis se observa el efecto de cada dimensión sobre las 
variables resultado, manteniendo constante la influencia de la otra dimensión. Para analizar el efecto de la $\mathrm{SDO}_{7}$ general, estimamos un factor de segundo orden construido a partir de las dimensiones SDO-D y SDO-A.

Ejecutamos los análisis en el software estadístico R 3.6.1 (R Development Core Team, 2019), con apoyo de los paquetes lavaan (Rosseel, 2012) y psych (Revelle, 2018). Los datos y materiales que utilizamos en estos estudios se encuentran disponibles en el siguiente enlace: https://osf.io/nxmwp/

\section{Figura 1}

Modelo de Ho et al. (2015) de Cuatro Factores (Conceptual X Método) en el que Cada Ítem Carga en un Factor Sustantivo y un Factor de Método

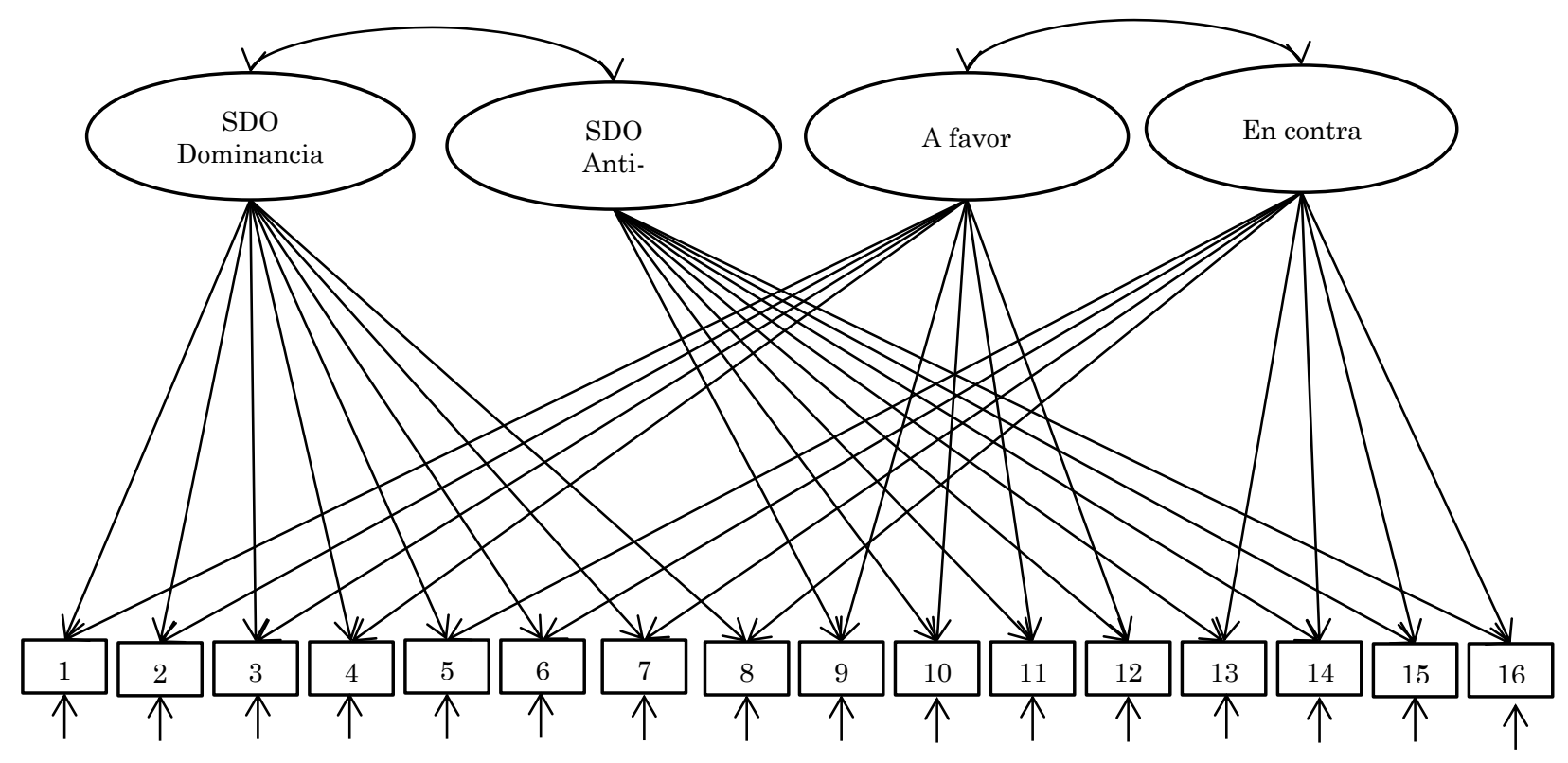

\section{Resultados}

\section{Análisis Descriptivo de la Escala de Orientación a la Dominancia Social}

En la Tabla 1 se puede observar que los ítems de la escala de la $\mathrm{SDO}_{7}$ suelen reportar valores relativamente bajos, considerando que los puntajes promedio se encuentran por debajo del punto medio de la escala $(3,5)$. En los dos estudios observamos que algunos de los ítems (i.e., dom6, dom8, anti-ig3, anti-ig5, anti-ig6 y anti-ig7) presentan un sesgo de respuesta hacia los valores más bajos de la escala y tienen poca variabilidad, lo que podría afectar su contribución a la escala. Inspeccionando el contenido de estos ítems, encontramos que cuatro de ellos son ítems invertidos y reflejan un rechazo a aceptar que los grupos desfavorecidos permanezcan en su situación y se les niegue oportunidades de desarrollo personal. En cuanto a la asociación de los ítems entre sí, encontramos que los ítems estuvieron asociados, en promedio, de forma positiva y estadísticamente significativa (Estudio 1, $r_{\text {promedio }}=0,26, D T=0,13$; Estudio 2, $r_{\text {promedio }}=0,27, \mathrm{DT}=0,11$; ver Tabla C del Anexo).

\section{Estructura Factorial de la Escala $\mathrm{SDO}_{7}$}

Los resultados sugieren que el Modelo 4 de cuatro factores, que estima las dimensiones sustantivas (SDO-D y SDO-A) y las dimensiones de método (ítems directos e inversos), obtuvo un ajuste adecuado a los datos, tanto para el Estudio 1 como para el Estudio 2. En ambos Estudios el modelo de cuatro factores obtuvo indicadores de ajuste sustantivamente mejores que los modelos que proponían una dimensión (Modelo 1), dos factores de método (Modelo 2) y dos factores sustantivos (Modelo 3) (ver Tabla 2). 
Tabla 1

Análisis Descriptivo de los Ítems de la Escala $\mathrm{SDO}_{7}$ Completa y por Dimensiones, para los Estudios 1 y 2

\begin{tabular}{|c|c|c|c|c|c|c|c|c|}
\hline \multirow{3}{*}{ Ítem } & \multicolumn{4}{|c|}{ Estudio $1(n=394)$} & \multicolumn{4}{|c|}{ Estudio $2(n=867)$} \\
\hline & \multicolumn{4}{|c|}{ Descriptivo } & \multicolumn{4}{|c|}{ Descriptivo } \\
\hline & $M$ & $D T$ & Asim & Curt & $M$ & $D T$ & Asim & Curt \\
\hline $\begin{array}{l}\text { Dom1. Una sociedad ideal requiere que algunos grupos de personas } \\
\text { están arriba y otros están abajo }\end{array}$ & 2,63 & 1,77 & 0,83 & $-0,43$ & 2,62 & 1,74 & 0,77 & $-0,63$ \\
\hline $\begin{array}{l}\text { Dom2. La dominación de unos grupos de personas sobre otros grupos } \\
\text { es un principio mediocre }(r)\end{array}$ & 3,19 & 1,99 & 0,52 & $-0,96$ & 2,99 & 1,92 & 0,62 & $-0,89$ \\
\hline $\begin{array}{l}\text { Dom3. Probablemente sea bueno que en la sociedad ciertos grupos de } \\
\text { personas están arriba y otros grupos de personas están abajo }\end{array}$ & 3,06 & 1,86 & 0,47 & $-0,94$ & 2,94 & 1,87 & 0,57 & $-0,98$ \\
\hline $\begin{array}{l}\text { Dom4. Algunos grupos de personas deberían ser mantenidos en su } \\
\text { posición social }\end{array}$ & 2,58 & 1,54 & 0,70 & $-0,26$ & 2,48 & 1,51 & 0,78 & $-0,28$ \\
\hline $\begin{array}{l}\text { Dom5. Algunos grupos de personas son simplemente inferiores a otros } \\
\text { grupos de personas }\end{array}$ & 2,12 & 1,64 & 1,33 & 0,61 & 2,13 & 1,65 & 1,40 & 0,81 \\
\hline $\begin{array}{l}\text { Dom6. Los grupos de personas en posiciones inferiores no tienen que } \\
\text { quedarse en su posición (r) }\end{array}$ & 1,91 & 1,32 & 1,99 & 4,28 & 1,73 & 1,23 & 2,52 & 7,04 \\
\hline Dom7. Ningún grupo de personas debería dominar en la sociedad (r) & 2,86 & 1,84 & 0,67 & $-0,66$ & 2,65 & 1,80 & 0,81 & $-0,57$ \\
\hline $\begin{array}{l}\text { Dom8. Los grupos de personas en posiciones inferiores son tan } \\
\text { merecedores como los grupos de personas en posiciones superiores (r) }\end{array}$ & 2,02 & 1,59 & 1,84 & 2,70 & 2,03 & 1,67 & 1,77 & 2,14 \\
\hline Anti-ig1. La igualdad entre grupos de personas debería ser nuestro ideal (r) & 2,24 & 1,50 & 1,18 & 0,62 & 2,33 & 1,60 & 1,34 & 0,99 \\
\hline Anti-ig2. Es injusto intentar igualar a los grupos de personas & 3,15 & 1,92 & 0,43 & $-0,95$ & 3,12 & 1,94 & 0,55 & $-0,96$ \\
\hline $\begin{array}{l}\text { Anti-ig3. Deberíamos trabajar para dar a todos los grupos de personas } \\
\text { igualdad de oportunidades para tener éxito }(\mathrm{r})\end{array}$ & 1,82 & 1,27 & 1,76 & 2,69 & 1,67 & 1,19 & 2,48 & 6,75 \\
\hline $\begin{array}{l}\text { Anti-ig4. La igualdad entre grupos de personas no debería ser nuestro } \\
\text { objetivo principal }\end{array}$ & 2,67 & 1,78 & 0,85 & $-0,30$ & 2,73 & 1,80 & 0,84 & $-0,43$ \\
\hline $\begin{array}{l}\text { Anti-ig5. No importa cuánto esfuerzo requiera, debemos luchar para } \\
\text { garantizar que todos los grupos de personas tengan las mismas } \\
\text { oportunidades en la vida (r) }\end{array}$ & 1,85 & 1,29 & 1,80 & 2,90 & 1,70 & 1,17 & 2,38 & 6,53 \\
\hline $\begin{array}{l}\text { Anti-ig6. No deberíamos promover la igualdad entre grupos de } \\
\text { personas }\end{array}$ & 1,96 & 1,47 & 1,74 & 2,48 & 1,97 & 1,49 & 1,80 & 2,63 \\
\hline $\begin{array}{l}\text { Anti-ig7. No deberíamos intentar garantizar que cada grupo de } \\
\text { personas tengan la misma calidad de vida }\end{array}$ & 1,82 & 1,37 & 1,93 & 3,33 & 1,78 & 1,40 & 2,28 & 4,72 \\
\hline $\begin{array}{l}\text { Anti-ig8. Deberíamos hacer todo lo posible para igualar las condiciones } \\
\text { de los diferentes grupos de personas (r) }\end{array}$ & 2,14 & 1,42 & 1,26 & 0,96 & 2,00 & 1,37 & 1,70 & 2,66 \\
\hline
\end{tabular}

Nota. $M=$ Media; $D T$ = Desviación típica; Asim = Asimetría; Curt = Curtosis.

Tabla 2

Estadísticos de Ajuste de los Modelos Estimados sobre la Estructura Factorial de la Escala de $\mathrm{SDO}_{7}$

\begin{tabular}{|c|c|c|c|c|c|c|}
\hline & Modelo & $\chi^{2}(g l)$ & RMSEA (90\% IC) & $\mathrm{CFI}_{\text {robusto }}$ & $\mathrm{TLI}_{\text {robusto }}$ & SRMR \\
\hline \multicolumn{7}{|c|}{ Estudio $1(n=394)$} \\
\hline Modelo 1 & Un factor & $366,269(104)^{* *}$ & $0,096(0,086-0,107)$ & 0,779 & 0,745 & 0,071 \\
\hline Modelo 2 & Dos factores (conceptual) & $337,862(103)^{* * *}$ & $0,091(0,081-0,102)$ & 0,803 & 0,770 & 0,069 \\
\hline Modelo 3 & Dos factores (método) & $271,902(103)^{* *}$ & $0,077(0,066-0,088)$ & 0,859 & 0,836 & 0,066 \\
\hline Modelo 4 & Cuatro factores (conceptual x método) & $141,947(90)^{* *}$ & $0,045(0,030-0,058)$ & 0,959 & 0,945 & 0,042 \\
\hline Modelo 5 & Cuatro factores (ajustado al contexto) & $70,973(44)^{*}$ & $0,046(0,025-0,064)$ & 0,973 & 0,959 & 0,041 \\
\hline \multicolumn{7}{|c|}{ Estudio $2(n=867)$} \\
\hline Modelo 1 & Un factor & $752,348(104)^{* *}$ & $0,097(0,090-0,103)$ & 0,783 & 0,750 & 0,066 \\
\hline Modelo 2 & Dos factores (conceptual) & $668,311(103)^{* *}$ & $0,091(0,084-0,097)$ & 0,812 & 0,781 & 0,064 \\
\hline Modelo 3 & Dos factores (método) & $510,031(103)^{* *}$ & $0,077(0,071-0,084)$ & 0,864 & 0,841 & 0,068 \\
\hline Modelo 4 & Cuatro factores (conceptual x método) & $165,374(86)^{* *}$ & $0,036(0,027-0,044)$ & 0,976 & 0,966 & 0,028 \\
\hline Modelo 5 & Cuatro factores (ajustado al contexto) & $63,091(40)^{*}$ & $0,027(0,013-0,040)$ & 0,992 & 0,986 & 0,021 \\
\hline
\end{tabular}

Nota. ${ }^{*} p<.05 ; * * p<.01 ; * * *<<.001$. 
Sin embargo, al inspeccionar las cargas factoriales del Modelo 4 en los dos estudios, encontramos que los ítems dom6 (Estudio 1: $b=0,026, p=0,672$; Estudio 2: $b=-0,43, p=0,452$ ) y dom8 (Estudio 1: $b=0,061$, $p=0,431$; Estudio 2: $b=0,026, p=0,607)$ no estuvieron asociados con la dimensión SDO-D. Del mismo modo, los ítems anti-ig3 (Estudio 1: $b=-0,371, p=0,175$; Estudio 2: $b=-0,265, p=0,139$ ) y anti-ig5 (Estudio 1: $b=-0,331$, $p=0,094$; Estudio 2: $b=-0,219, p=0,230$ ) no estuvieron asociados con la dimensión SDO-A, además de que los coeficientes fueron negativos. Estos cuatro ítems, que están redactados en sentido negativo (contrait), representan el rechazo a aceptar que los grupos de personas desfavorecidas tengan que permanecer en su posición y que no se les brinde oportunidades de desarrollo. Además, los estadísticos descriptivos de estos ítems sugieren una baja variabilidad de respuestas y un sesgo para responder hacia los valores más bajos de la escala.

Teniendo en cuenta la información anterior y con el fin de adaptar los ítems y el constructo al contexto colombiano, decidimos evaluar un último modelo, eliminando estos ítems que no presentaron propiedades adecuadas en las muestras de los Estudios 1 y 2 (i.e., dom6, dom8, anti-ig3 y anti-ig5). Los resultados indicaron que el modelo adaptado, incluyendo dos factores sustantivos y dos de método, tuvo un ajuste apropiado tanto para el Estudio 1 como para el Estudio 2 (ver Tabla 2). Comparamos el cambio en los indicadores de ajuste para determinar cualitativamente el modelo que mostró mejor ajuste. Al comparar el modelo adaptado al contexto colombiano (Modelo 5) con el hipotetizado originalmente (Modelo 4), encontramos que el Modelo 5 presentó un ajuste sustantivamente mejor que el Modelo 4 en los dos estudios (Estudio 1: $\Delta \mathrm{CFI}=0,014, \Delta \mathrm{AIC}=-4375,10, \Delta \mathrm{BIC}=-4421,12 ;$ Estudio 2: $\Delta \mathrm{CFI}=0,016, \Delta \mathrm{AIC}=-10298,06$, $\left.\Delta \mathrm{BIC}=-10354,54^{2}\right)($ ver Figura 2 para las cargas factoriales en los dos estudios).

\section{Figura 2}

Modelo Final Adaptado al Contexto Colombiano con las Cargas Factoriales en los Estudios 1 y 2 (Estudio 1/Estudio 2)

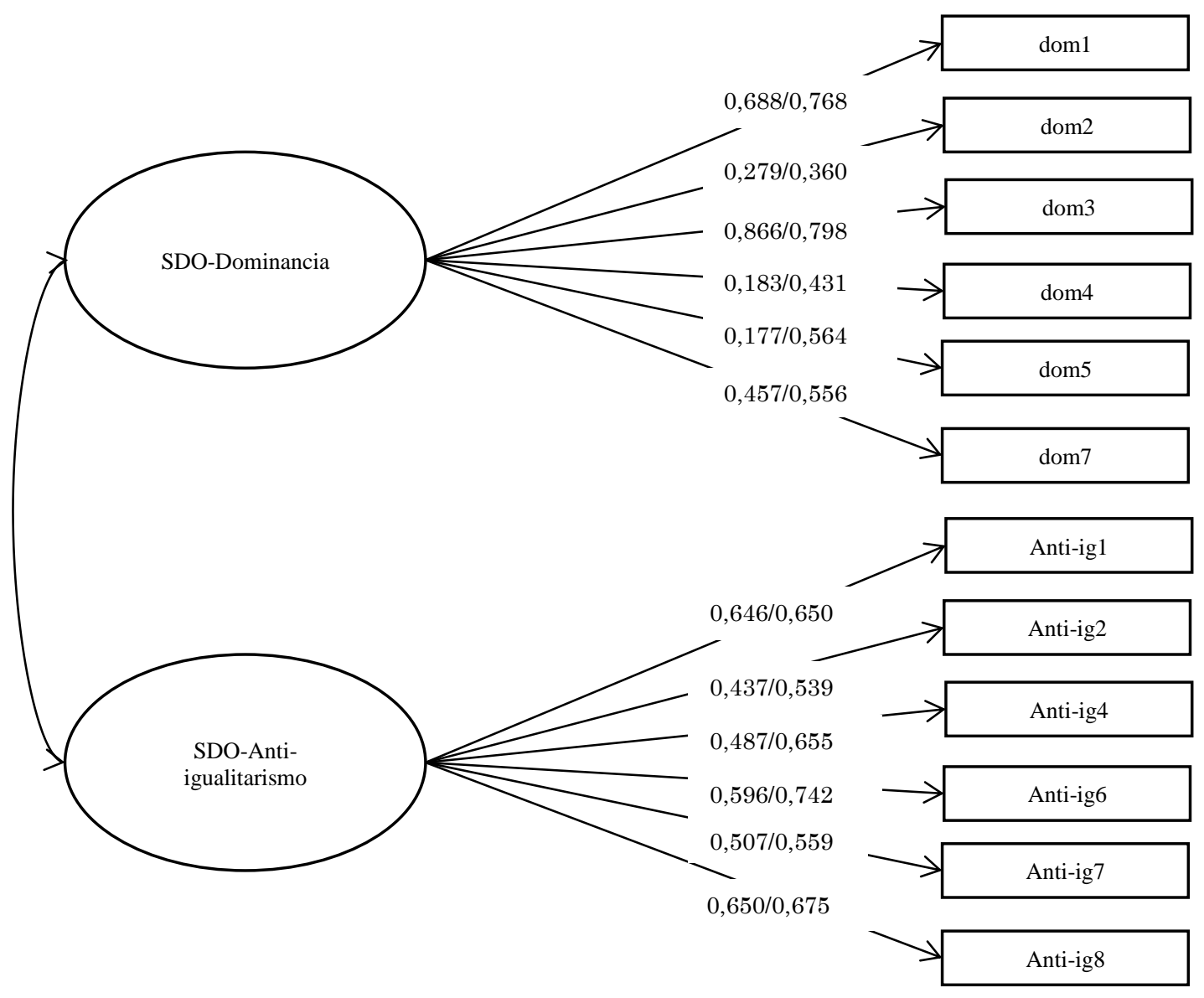

Nota. Estimamos los factores de método, pero no los presentamos en la figura para facilitar su lectura. 


\section{Análisis de Consistencia Interna de la Escala $\mathrm{SDO}_{7}$ y sus Dimensiones}

Luego de determinar el modelo final (Modelo 5), examinamos la consistencia interna y los estadísticos descriptivos de la escala. Las dimensiones de la escala mostraron indicadores de consistencia interna apropiados según los parámetros usados comúnmente en la validación de escalas (Carretero-Dios \& Pérez, 2007). Alfa de Cronbach (a) y Omega de MacDonald ( $\omega$ ) tuvieron valores por encima de 0,70, tanto en la dimensión SDO-D ( $\left.\alpha_{\text {Estudio } 1}=0,73 ; \alpha_{\text {Estudio } 2}=0,80 ; \omega_{\text {Estudio }}=0,72 ; \omega_{\text {Estudio } 2}=0,77\right)$, como en la dimensión SDO-A $($ a Estudio $1=0,80 ;$ astudio $2=0,79 ; \omega$ Estudio1 $=0,79 ; \omega$ Estudio $=0,80)$. Además, la correlación ítem-total mostró que

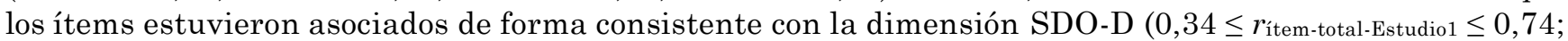
$\left.0,39 \leq r_{\text {item-total-Estudio } 2} \leq 0,77\right)$ y con la dimensión SDO-A $\left(0,50 \leq r_{\text {ítem-total-Estudio1 }} \leq 0,71 ; 0,49 \leq r_{\text {ítem-total-Estudio } 2} \leq 0,72\right)$. Así, los indicadores anteriores sugieren que los ítems de la escala presentan evidencias de consistencia interna apropiadas para medir las dimensiones de la $\mathrm{SDO}_{7}$.

A nivel descriptivo, los resultados de una prueba $t$ para muestras apareadas indican que la media de los ítems de SDO-D (Estudio 1: $M=2,75, D T=1,15$; Estudio $2: M=2,62, D T=1,16$ ) fueron mayores que la media de los de SDO-A (Estudio 1: $M=2,33, D T=1,12$; Estudio 2: $M=2,29, D T=1,11$ ), tanto en el Estudio 1, $t(360)=7,46, p<0,001, d=0,39,95 \%$ IC $[0,29,0,50]$, como en el Estudio $2, t(721)=8,44, p<0,001, d=0,31$, $95 \%$ IC $[0,24,0,39]$. Las diferencias de medias las estimamos usando las variables observables y no las variables latentes, debido a que las medias de las variables latentes no son interpretables de forma directa y sería necesario imponer restricciones adicionales al modelo de medición. Por tanto, decidimos usar las variables observables por ser un procedimiento estandarizado en este tipo de estudios (i.e., prueba $t$ ), puesto que permite conservar la métrica original de la escala y sus resultados pueden interpretarse directamente.

\section{Prueba de Invarianza por Sexo}

Estimamos el modelo final adaptado para el contexto colombiano con dos factores sustantivos (conceptuales) y fuimos adicionando restricciones para evaluar la bondad de ajuste de los modelos subsecuentes (Hirschfeld \& von Brachel, 2014). Decidimos usar este modelo de dos factores sustantivos y no estimar los factores de método por ser más parsimonioso para representar las dimensiones de la SDO 7 , puesto que el modelo final (Modelo 5) de cuatro factores tiene más parámetros para estimar y presentó problemas de convergencia.

La invarianza configural obtuvo indicadores de ajuste apropiados (Brown, 2015), lo que quiere decir que la estructura de los factores fue similar en hombres y mujeres (ver Tabla 3). En cuanto a la invarianza métrica, encontramos que el modelo no redujo su bondad de ajuste, siguiendo el criterio de $\Delta C F I<0,01$ sugerido en la literatura (Cheung \& Rensvold, 2002; Kline, 2016). Siguiendo este mismo criterio, encontramos que también se retuvo la invarianza escalar y la residual, por lo que cabría esperar que los interceptos de los ítems y los errores residuales sean equivalentes entre grupos. Estos resultados son corroborados con la prueba del cambio en $\chi^{2}$ (log-likelihood ratio), la que sugiere que los modelos con mayores restricciones no tuvieron diferencias en su bondad de ajuste (Configural versus Métrica, $\Delta \chi^{2}=11,910, \Delta g l=10, p=0,291$; Métrica versus Escalar, $\Delta \chi^{2}=12,944, \Delta g l=10, p=0,226$; Residual versus Escalar, $\Delta \chi^{2}=15,001, \Delta g l=12, p=0,241$ ). En este sentido, podemos afirmar que la escala de $\mathrm{SDO}_{7}$ presenta propiedades psicométricas equivalentes para hombres y mujeres en lo que respecta a la estructura factorial, las cargas factoriales, los interceptos y los errores residuales de los ítems.

Tabla 3

Prueba de Invarianza entre Grupos por Sexo

\begin{tabular}{|c|c|c|c|c|c|c|c|}
\hline Invarianza & $\chi^{2}(g l)$ & RMSEA (90\% IC) & $\mathrm{SRMR}_{\mathrm{R}}$ & $\mathrm{TLI}_{\mathrm{R}}$ & $\mathrm{CFI}_{\mathrm{R}}$ & $\Delta \mathrm{CFI}$ & Invarianza \\
\hline 1. Configural ${ }^{a}$ & $241,037(106)^{*}$ & $0,066(0,055,0,077)$ & 0,054 & 0,919 & 0,935 & - & - \\
\hline 2. Métricab & $252,113(116)^{*}$ & $0,063(0,053,0,070)$ & 0,059 & 0,925 & 0,934 & 0,001 & Sí \\
\hline 3. Escalar ${ }^{\mathrm{c}}$ & $266,434(126)^{*}$ & $0,061(0,051,0,071)$ & 0,061 & 0,930 & 0,933 & 0,001 & Sí \\
\hline 4. Estricta ${ }^{\mathrm{d}}$ & $274,131(138)^{*}$ & $0,060(0,049,0,070)$ & 0,063 & 0,933 & 0,930 & 0,003 & Sí \\
\hline
\end{tabular}

Nota. R: estadísticos de ajuste son robustos; a: patrón de las cargas factoriales similares en los factores; b: equivalencia en las cargas factoriales; c: equivalencia de los interceptos; d: equivalencia de las varianzas residuales. 
El análisis de un modelo MIMIC arrojó que las mujeres - comparadas con los hombres- reportarían 0,328 unidades menos en la puntuación de la variable latente de SDO-D $(b=-0,328, E E=0,115, p=0,004)$ y $0,267$ unidades menos en la escala de SDO-A ( $b=-0,285, E E=0,086, p=0,001)$. En cuanto a la influencia del estatus socioeconómico, encontramos una asociación inversa entre el nivel educacional y los niveles de SDO-D $(b=-0,254, p<0,001)$ y de SDO-A $(b=-0,132, p=0,008)$, pero el estatus socioeconómico por ingresos no estuvo asociado con ninguna de las dimensiones (SDO-D: $b=0,036, p=0,300$; SDO-A: $b=-0,004$, $E E p=0,870)$. El estatus subjetivo, por su parte, estuvo asociado positivamente con la dimensión de SDO-D $(b=0,098, p=0,034)$, pero no con la de SDO-A $(b=0,052, p=0,100)$.

\section{Validez Predictiva de la $\mathrm{SDO}_{7}$ y sus Dimensiones de Dominancia y Anti-Igualitarismo}

Estos modelos los estimamos solo con los factores sustantivos (SDO-D y SDO-A) que incluyen los ítems finales de la escala, puesto que, al incluir los factores de método (pro-trait y con-trait), el modelo presentaba problemas de convergencia e identificación por causa de su complejidad. Sin embargo, el modelo con dos factores sustantivos adaptado al contexto colombiano es más parsimonioso y presentó indicadores de ajuste apropiados tanto en el Estudio $1, \chi^{2}(53, n=394)=109,978, p<0,001, \mathrm{RMSEA}=0,063,90 \%$ IC $[0,046,0,080]$, $\mathrm{CFI}_{\text {robusto }}=0,937, \mathrm{TLI}_{\text {robusto }}=0,922, \mathrm{SRMR}=0,053$, como en el Estudio $2, \chi^{2}(53, n=867)=190,655, p<0,001$, RMSEA $=0,061,90 \%$ I $[0,052,0,070], \mathrm{CFI}_{\text {robusto }}=0,946, \mathrm{TLI}_{\text {robusto }}=0,933, \mathrm{SRMR}=0,053$. Por tal motivo, como sugiere la literatura (Brown, 2015), decidimos utilizar el modelo más simple para estimar las asociaciones entre variables en el modelo final.

Como se puede observar en la Tabla 4, la escala general de la $\mathrm{SDO}_{7}$ tuvo un efecto directo en la ideología política y la justificación del sistema económico, es decir, a mayor puntaje en $\mathrm{SDO}_{7}$ mayor posicionamiento hacia la derecha en el espectro político, y una mayor justificación del sistema económico, y un efecto inverso en patriotismo, es decir, a mayor puntaje en $\mathrm{SDO}_{7}$ menores niveles de patriotismo. En relación con el apoyo a medidas sociales y políticas, la $\mathrm{SDO}_{7}$ tuvo un efecto negativo sobre el apoyo a la protesta social, la preocupación por la desigualdad económica, la redistribución de recursos, el matrimonio homosexual, la inversión social y la inclusión educativa. También tuvo un efecto inverso sobre el apoyo a valores sociales, como la paz, la justicia y la igualdad. Por otra parte, la $\mathrm{SDO}_{7}$ general tuvo un efecto positivo sobre el apoyo a la solución militar del conflicto, la pena de muerte, el castigo físico como forma de educar a los hijos, la justificación de la desigualdad y la valoración de la riqueza.

En cuanto a la validez predictiva de las dimensiones de la $\mathrm{SDO}_{7}$, observamos semejanzas y diferencias importantes. Dentro de las semejanzas, la SDO-D y la SDO-A tuvieron efectos positivos en la ideología política en el Estudio 2, pero no en el Estudio 1. Asimismo, la $\mathrm{SDO}_{7}$ general tuvo un efecto negativo sobre el apoyo a la protesta social y sobre el apoyo a la redistribución en los dos estudios.

Entre las diferencias sustantivas, hubo efectos de las dimensiones de la $\mathrm{SDO}_{7}$ en las variables ideológicas y actitudinales. Particularmente, en el Estudio 2, la SDO-D tuvo un efecto positivo en la justificación del sistema económico, pero no el SDO-A. Del mismo modo, en el Estudio 2, la SDO-D tuvo un efecto positivo sobre el apoyo a la solución militar del conflicto, pero no el SDO-A, mientras que tuvo un efecto inverso sobre la preocupación por la desigualdad económica, pero no el SDO-A. La SDO-D también tuvo un efecto positivo sobre el apoyo a la inversión social y sobre valores como la autoridad, la riqueza, la influencia, la riqueza y el respeto a los padres.

Adicionalmente, en el Estudio 1 encontramos que algunas variables actitudinales y valores sociales fueron predichas exclusivamente por alguna dimensión. Por ejemplo, la SDO-D (y no el SDO-A) tuvo un efecto positivo sobre el apoyo al castigo físico como forma de educar, apreciar la riqueza como valor y honrar a los padres. Por otro lado, el SDO-A (y no la SDO-D) tuvo un efecto positivo sobre la justificación de la desigualad y un efecto negativo sobre el apoyo a la inversión social y la inclusión educativa, así como sobre el apoyo a valores sociales, como la autoridad, la influencia, la paz, la justicia, la igualdad y la disciplina. 
Tabla 4

Efectos de las Variables Latentes de $\mathrm{SDO}_{7}$ (General y Dimensiones) en Ideologías, Apoyo a Medidas Sociales y Políticas y Valores Sociales (Estudios 1 y 2)

\begin{tabular}{|c|c|c|c|c|c|c|c|c|c|c|c|c|}
\hline & \multicolumn{6}{|c|}{ Estudio 1} & \multicolumn{6}{|c|}{ Estudio 2} \\
\hline & \multicolumn{2}{|c|}{$\mathrm{SDO}_{7}$ (general) } & \multicolumn{2}{|c|}{ Dominancia } & \multicolumn{2}{|c|}{ Anti-igualitarismo } & \multicolumn{2}{|c|}{$\mathrm{SDO}_{7}$ (general) } & \multicolumn{2}{|c|}{ Dominancia } & \multicolumn{2}{|c|}{ Anti-igualitarismo } \\
\hline & $B$ & $95 \%$ IC & $B$ & $95 \%$ IC & $B$ & $95 \%$ IC & $B$ & $95 \%$ IC & $B$ & $95 \%$ IC & $B$ & $95 \%$ IC \\
\hline \multicolumn{13}{|c|}{ Ideologías } \\
\hline Ideología política & $0,19^{*}$ & {$[0,01,0,37]$} & 0,15 & {$[-0,08,0,31]$} & 0,05 & {$[-0,20,0,29]$} & $0,30^{* * *}$ & {$[0,23,0,46]$} & $0,18^{* *}$ & {$[0,05,0,25]$} & $0,12^{*}$ & {$[0,00,0,25]$} \\
\hline $\begin{array}{l}\text { Justificación del sistema } \\
\text { económico }\end{array}$ & $0,22^{* *}$ & {$[0,09,0,47]$} & 0,24 & {$[-0,08,0,54]$} & 0,00 & {$[-0,36,0,36]$} & $0,25^{* * *}$ & {$[0,21,0,49]$} & $0,34 * * *$ & {$[0,22,0,46]$} & $-0,08$ & {$[-0,25,0,05]$} \\
\hline Patriotismo & - & - & - & - & - & - & $-0,14^{* *}$ & {$[-0,32,-0,05]$} & 0,02 & {$[-0,10,0,14]$} & $-0,16^{*}$ & {$[-0,36,-0,02]$} \\
\hline \multicolumn{13}{|c|}{ Apoyo a medidas sociales y políticas } \\
\hline Protesta social & $-0,38 * * *$ & {$[-0,83,-0,31]$} & 0,06 & {$[-0,14,0,27]$} & $-0,42^{* * *}$ & {$[-0,98,-0,26]$} & $-0,33^{* * *}$ & {$[-0,69,-0,37]$} & $-0,17 * *$ & {$[-0,36,-0,05]$} & $-0,15^{*}$ & {$[-0,42,-0,03]$} \\
\hline Solución militar al conflicto & $0,21^{* *}$ & {$[0,12,0,68]$} & 0,04 & {$[-0,20,0,32]$} & 0,17 & {$[-0,05,0,66]$} & $0,30 * * *$ & {$[0,29,0,60]$} & $0,20^{* *}$ & {$[0,07,0,35]$} & 0,10 & {$[-0,04,0,33]$} \\
\hline $\begin{array}{l}\text { Preocupación por la } \\
\text { desigualdad }\end{array}$ & $-0,28^{* * *}$ & {$[-0,59,-0,17]$} & $-0,14$ & {$[-0,33,0,05]$} & $-0,14$ & {$[-0,45,0,08]$} & $-0,25^{* * *}$ & {$[-0,32,-0,15]$} & $-0,14^{*}$ & {$[-0,19,-0,01]$} & $-0,11$ & {$[-0,21,0,02]$} \\
\hline $\begin{array}{l}\text { Redistribución (diferentes } \\
\text { medidas) }\end{array}$ & $-0,40 * * *$ & {$[-1,01,-0,49]$} & $-0,24^{* *}$ & {$[-0,56,-0,07]$} & $-0,17^{*}$ & {$[-0,64,0,01]$} & $-0,51^{* * *}$ & {$[-0,56,-0,38]$} & $-0,23 * * *$ & {$[-0,23,-0,08]$} & $-0,27 * * *$ & {$[-0,34,-0,12]$} \\
\hline Pena de muerte & $0,16^{*}$ & {$[0,07,0,67]$} & 0,05 & {$[-0,20,0,36]$} & 0,11 & {$[-0,14,0,63]$} & - & - & - & - & - & - \\
\hline Penas más drásticas & - & - & & - & - & - & 0,08 & {$[-0,02,0,29]$} & 0,10 & {$[-0,03,0,28]$} & $-0,02$ & {$[-0,23,0,17]$} \\
\hline Matrimonio homosexual & $-0,21^{* *}$ & {$[-0,78,-0,15]$} & $-0,16$ & {$[-0,57,0,05]$} & $-0,05$ & {$[-0,53,0,30]$} & - & - & - & - & - & - \\
\hline Castigo físico a niños/as & $0,21^{* *}$ & {$[0,17,0,71]$} & $0,20 *$ & {$[0,04,0,56]$} & 0,02 & {$[-0,31,0,39]$} & - & - & - & - & - & - \\
\hline $\begin{array}{l}\text { Justificación de la } \\
\text { desigualdad }\end{array}$ & $0,34^{* * *}$ & {$[0,42,0,93]$} & 0,13 & {$[-0,05,0,43]$} & $0,21^{* *}$ & {$[0,09,0,72]$} & - & - & - & - & - & - \\
\hline Inversión social & $-0,23 * * *$ & {$[-0,69,-0,16]$} & $0,23^{*}$ & {$[0,05,0,54]$} & $-0,42^{* * *}$ & {$[-1,15,-0,37]$} & - & - & - & - & - & - \\
\hline Inclusión educativa & $-0,32^{* * *}$ & {$[-0,82,-0,28]$} & $-0,00$ & {$[-0,25,0,24]$} & $-0,31^{* *}$ & {$[-0,91,-0,12]$} & - & - & - & - & - & - \\
\hline \multicolumn{13}{|c|}{ Valores sociales } \\
\hline Autoridad & 0,09 & {$[-0,21,0,56]$} & $0,57^{* * *}$ & {$[0,40,1,29]$} & $-0,43 * * *$ & {$[-1,35,-0,24]$} & - & - & - & - & - & - \\
\hline Influencia & 0,02 & {$[-0,35,0,42]$} & $0,56 * * *$ & {$[0,39,1,25]$} & $-0,49 * * *$ & {$[-1,44,-0,37]$} & - & - & - & - & - & - \\
\hline Riqueza & $0,18^{*}$ & {$[0,02,0,59]$} & $0,32^{*}$ & {$[0,04,0,79]$} & $-0,12$ & {$[-0,64,0,26]$} & - & - & - & - & - & - \\
\hline $\mathrm{Paz}$ & $-0,49 * * *$ & {$[-1,01,-0,41]$} & 0,10 & {$[-0,16,0,37]$} & $-0,56^{* * *}$ & {$[-1,21,-0,38]$} & - & - & - & - & - & - \\
\hline Justicia & $-0,48^{* * *}$ & {$[-0,82,-0,32]$} & 0,05 & {$[-0,17,0,27]$} & $-0,51^{* * *}$ & {$[-0,92,-0,26]$} & - & - & - & - & - & - \\
\hline Igualdad & $-0,65 * * *$ & {$[-1,33,-0,63]$} & $-0,07$ & {$[-0,36,0,20]$} & $-0,57 * * *$ & {$[-1,32,-0,35]$} & - & - & - & - & - & - \\
\hline Honrar a padres & $-0,03$ & {$[-0,36,0,26]$} & $0,26^{*}$ & {$[-0,01,0,70]$} & $-0,27$ & {$[-0,93,0,03]$} & - & - & - & - & - & - \\
\hline Disciplina & $-0,14$ & {$[-0,56,0,09]$} & 0,22 & {$[-0,03,0,61]$} & $-0,33^{* *}$ & {$[-0,97,-0,13]$} & - & - & - & - & - & - \\
\hline
\end{tabular}

Nota. $B$ = Coeficiente estandarizado; *** $p<0,001 ; * * p<0,01 ; * p<0,05$ 


\section{Discusión}

$\mathrm{El}$ objetivo de nuestra investigación fue adaptar y validar la escala $\mathrm{SDO}_{7}$ al castellano en una muestra colombiana. En cuanto a la primera hipótesis de trabajo, encontramos que la estructura factorial propuesta en la versión de $\mathrm{SDO}_{7}$ (Ho et al., 2015) tuvo indicadores de ajuste global apropiados según algunos criterios usados en la literatura (Brown, 2015), lo cual sugiere que el constructo de SDO se representa apropiadamente a través de dos factores sustantivos y dos factores de método. Sin embargo, a pesar de obtener indicadores de ajuste global apropiados, hallamos fuentes de desajuste local en cuatro ítems que plantearon la necesidad de hacer modificaciones adicionales para adaptarse correctamente al contexto colombiano. Los ítems eliminados hacían referencia al rechazo a brindar oportunidades de desarrollo y de ascenso social, lo cual va en contra del ideal meritocrático y de superación, que es bastante aceptado y promovido en el contexto colombiano (García-Sánchez et al., 2018). Por tanto, estos elementos fueron eliminados de la escala, por considerar que no se adaptan bien al contexto colombiano.

Adicionalmente, los indicadores de confiabilidad de la escala sugieren que los ítems estuvieron asociados consistentemente y con las dimensiones esperadas. La validez predictiva también fue apoyada por nuestros resultados, en tanto que el constructo general de SDO como sus dimensiones latentes de SDO-D y SDO-A se asociaron consistentemente con otras variables ideológicas y actitudinales que mantienen y promueven la desigualdad. Por tanto, consideramos que la escala de $\mathrm{SDO}_{7}$ adaptada al contexto colombiano cumple con los criterios psicométricos necesarios para ser utilizada en este.

En relación a la hipótesis de trabajo $\mathrm{H}_{2}$, sobre las diferencias en SDO por sexo, observamos que las mujeres reportaron menores niveles de SDO, tanto en las dimensiones latentes de SDO-D como de SDO-A. Este resultado confirma uno de los postulados de la TDS que plantea que los grupos que se encuentran en posiciones aventajadas, de privilegio y/o poder (e.g., los hombres en sociedades patriarcales) suelen reportar mayores niveles de SDO (Pratto et al., 1994; Sidanius et al., 2000, 2017).

En cuanto a la Hipótesis $\mathrm{H}_{3}$, advertimos evidencia parcial en relación con el efecto positivo del estatus sociodemográfico sobre la SDO. Por un lado, en el Estudio 2, el estatus objetivo por ingresos no estuvo asociado con las dimensiones de la SDO, pero el estatus objetivo por nivel educativo sí tuvo un efecto negativo sobre las dos dimensiones. Es decir, cuanto mayor nivel educativo de las personas, menor fueron sus niveles de SDO-D y SDO-A. Aunque las personas de mayor nivel educativo también poseen un mayor estatus social, que bien podría motivarlas a apoyar la desigualdad, el estatus por educación también acarrea un proceso de socialización política que promueve valores sociales (e.g., equidad, respeto, dignidad) que podrían reducir la disposición a favorecer la dominancia y desigualdad.

Por otra parte, encontramos que el estatus subjetivo estuvo asociado positivamente con SDO-D, pero no con SDO-A. Estos resultados corroboran lo planteado por la teoría, puesto que las jerarquías sociales por categorías arbitrarias (e.g., dinero, estatus económico) dependen más de las características del contexto que las categorías biológicas (i.e., sexo) (Sidanius et al., 2000). Además, estos resultados confirman algunos planteamientos sobre la relación positiva del estatus socioeconómico con la motivación a justificar el estatus quo y legitimar la desigualdad (Friesen et al., 2019; Jost, 2019). Así, nuestros resultados sugieren que la percepción subjetiva del propio estatus social podría estar asociada con los niveles de SDO-D y SDO-A, independientemente de los ingresos económicos objetivos. Esta idea ha sido corroborada en otros estudios que señalan que la percepción subjetiva del estatus es un mecanismo clave en la legitimación de la desigualdad y la dominación entre grupos (Brown-Iannuzzi et al., 2015, 2021). Por tanto, el supuesto teórico que plantea que las personas en posiciones de mayor privilegio y poder tenderían a reportar mayores niveles de SDO necesita ser puesta en el contexto sobre lo que implica tener más estatus y privilegio. Sidanius et al. (2000) plantean que el estatus socioeconómico podría interactuar con la estructura del contexto social y mostrar matices a la hora de relacionarse con la SDO. En algunos contextos, las personas con mayor estatus social y poder son las mismas que tienen acceso a información y espacios de socialización que les permiten desafiar el estatus quo. Asimismo, es plausible que no se trate solamente de cuánto poder material tienen las personas, sino de la percepción subjetiva que tienen respecto de su posición social superior. Futuras investigaciones podrían explorar los diferentes matices que tiene esta relación entre el estatus social y la SDO.

Adicionalmente, las dimensiones de la $\mathrm{SDO}_{7}$ mostraron tener validez predictiva sobre ideologías y actitudes políticas que justifican la desigualdad de forma diferenciada, tal como planteamos en las hipótesis $\mathrm{H}_{4 a}$ y $\mathrm{H}_{4 b}$. Así, encontramos evidencia a favor de la $\mathrm{H}_{4 a}$, en tanto que la dimensión de SDO-D estuvo asociada principalmente con acciones que promueven explícitamente el sometimiento y la dominación de otros grupos, 
tales como el apoyo a la solución militar del conflicto armado en Colombia, el rechazo al matrimonio homosexual, la aceptación del castigo físico a los niños y la valoración de la autoridad, la influencia y la riqueza. Estas medidas dejan entrever la aprobación del uso legítimo de la violencia (e.g., el caso del conflicto armado, el castigo físico a los niños), del prejuicio (en el caso del matrimonio igualitario), o de la adhesión a valores que promueven relaciones de poder (e.g., autoridad, disciplina).

Por otra parte, también confirmamos la $\mathrm{H}_{4 b}$, puesto que la dimensión de SDO-A estuvo asociada principalmente con acciones que perpetúan dinámicas institucionales o sociales que mantienen las desigualdades entre grupos, tales como el rechazo a la protesta social, la inversión social, la inclusión educativa, la redistribución de recursos, así como una menor adherencia a valores como la paz, la justicia y la igualdad. En este sentido, el SDO-A es un antecedente de las actitudes hacia políticas públicas o medidas sociales que mantienen el estatus quo y las relaciones de desigualdad entre grupos. Por ejemplo, desaprobar la protesta social es una forma de aceptar el estatus quo o de no apoyar la redistribución de recursos (económicos, sociales, educativos, entre otros). Por tanto, es importante considerar que la SDO-D y el SDO-A, aunque conforman el mismo constructo de SDO, encierran diferentes motivaciones y formas de mantener/promover las relaciones de poder y desigualdad. Nuestros resultados confirman el planteamiento de Ho et al. (2015) sobre los diferentes efectos de la $\mathrm{SDO}_{7}$ sobre actitudes sociales.

No obstante, algunas variables no estuvieron asociadas con las dimensiones de la SDO en la forma en que habíamos hipotetizado. Por ejemplo, en el Estudio 2, la SDO-D (pero no la SDO-A) estuvo asociada positivamente con la justificación del sistema económico y negativamente con la preocupación por la desigualdad. De forma semejante, la SDO-D estuvo negativamente asociada con el apoyo a la protesta social (Estudio 2) y la redistribución de recursos (Estudios 1 y 2). Aunque nuestros resultados podrían parecer inesperados a la luz de la literatura (e.g., Ho et al., 2015; Jost et al., 2003), hay tres aspectos que podrían explicar estos hallazgos. Primero, las dimensiones de $\mathrm{SDO}_{7}$ son dos factores que reflejan matices del mismo constructo, por tanto, su diferenciación puede resultar difícil de determinar en algunos estudios empíricos. Segundo, es posible que haya diferencias en la forma de interpretar los constructos según las características de la muestra y el contexto de aplicación de la escala. En Colombia, la desigualdad social se ha mantenido estrechamente ligada al ejercicio de la violencia política (i.e., conflicto armado interno), de modo que la subordinación de los grupos desfavorecidos ha sido facilitada por el ejercicio sistemático de la violencia por parte de diferentes actores armados (e.g., grupos guerrilleros, paramilitares, bandas criminales). Por tanto, el uso de la violencia explícita — asociada a la dominancia - se ha utilizado como una estrategia para mantener relaciones de desigualdad entre grupos sociales (e.g., desplazamientos forzados de campesinos, expropiación de tierras, amenazas a grupos de oposición que representan minorías étnicas). Así, la justificación del sistema económico, el apoyo a la protesta social y la tolerancia a la desigualdad podrían reflejar no solo una mayor aceptación de la desigualdad institucionalizada, sino también el vínculo entre desigualdad y violencia que se aprecia en el contexto histórico, social y político del país.

Tercero, es posible que las diferencias puedan estar relacionadas con el significado de la escala en el contexto colombiano. Es decir, algunos indicadores de SDO-D y SDO-A entran en conflicto con algunos ideales culturales (e.g., la meritocracia). En este sentido, es posible que la SDO refleje una disposición a mantener la dominancia y la desigualdad, pero dentro de un contexto donde las personas puedan tener algún grado de movilidad social. Estas ideas podrían ser exploradas en profundidad en futuras investigaciones, en las que se repliquen nuestros resultados y se analice la relación de la SDO con las ideas de la meritocracia y la expectativa de movilidad social.

Adicionalmente, nuestros resultados mostraron una asociación negativa entre SDO-A y patriotismo, cuando se hubiera esperado que esta asociación sucediera con la SDO-D (Cárdenas et al., 2010). La literatura sugiere que las personas más autoritarias tienden a ser más conservadoras y aceptar más la autoridad (Janos et al., 2018; Ungaretti \& Etchezahar, 2016) y, por tanto, apoyarían más la desigualdad y el estatus quo. Sin embargo, la recolección de información para nuestros estudios tuvo lugar en un momento político de alta impopularidad del gobierno y polarización política como consecuencia del proceso de paz. Estos factores pudieron haber reforzado la desidentificación de las personas con el gobierno. Por tanto, cuanto mayor SDO, mayor rechazo hacia la gestión del gobierno, que en su momento promovía los diálogos de paz en los que se discutían las políticas de redistribución de recursos y reducción de las desigualdades.

En cuanto a las limitaciones de nuestro estudio, es importante señalar varios aspectos. Primero, los/as participantes de esta investigación fueron en su mayoría estudiantes universitarios. Aunque los/as participantes provienen de un sector bastante diverso de la población (i.e., diferentes estratos económicos y 
zonas del país), siguen siendo un grupo reducido de personas que acceden a la educación superior. El Estudio 2 incluyó una población más diversa, pero esta comunidad sigue compartiendo algunos valores sociales progresistas y/o liberales que hacen parte de la cultura organizacional de una universidad pública latinoamericana. Por tanto, es necesario replicar nuestros resultados iniciales a través de investigaciones con grupos de población general, que ayuden a corroborar algunas de las tendencias que hemos reportado.

Otra limitación tiene que ver con la falta de escalas de medición adaptadas y validadas en Colombia sobre temas sociales y políticos, lo cual nos dificultó usar mejores medidas para la evaluación de la validez predictiva de la SDO. Esta situación nos llevó a usar medidas basadas en indicadores de encuestas de uno o pocos ítems, lo cual puede afectar la relación de tales indicadores con la SDO. En este sentido, es importante usar en futuros estudios otras medidas con mejores propiedades psicométricas.

Finalmente, para utilizar la escala, es posible estimar tanto los factores sustantivos como los de método, tal como lo especificamos en el Modelo 5. Sin embargo, este modelo implica la especificación de un modelo más complejo que puede acarrear problemas de convergencia. Por tanto, la escala también podría usarse estimando los dos factores sustantivos y controlando la correlación de los errores residuales de los ítems invertidos. Estimamos que ambas opciones son viables y representan apropiadamente el constructo, aunque la segunda opción es más parsimoniosa. Además, nuestros resultados sugieren que la SDO-D podría usarse más para estudiar actitudes hacia medidas más explícitas, hostiles o de confrontación que mantienen la jerarquía social, mientras que la dimensión de SDO-A sería más apropiada para analizar el apoyo a estrategias más sutiles e institucionalizadas que mantienen las desigualdades sociales.

\section{Referencias}

Altemeyer, B. (1998). The other "authoritarian personality". Advances in Experimental Social Psychology, 30, 47-92. https://doi.org/10.1016/S0065-2601(08)60382-2

Brown-Iannuzzi, J. L., Lundberg, K. B., Kay, A. C. \& Payne, B. K. (2015). Subjective status shapes political preferences. Psychological Science, 26(1), 15-26. https://doi.org/10.1177/0956797614553947

Brown-Iannuzzi, J. L., Lundberg, K. B., Kay, A. C. \& Payne, B. K. (2021). A privileged point of view: Effects of subjective socioeconomic status on naïve realism and political division. Personality and Social Psychology Bulletin, 47(2), 241-256. https://doi.org/10.1177/0146167220921043

Brown, T. A. (2015). Confirmatory factor analysis for applied research: Second edition. Guilford Press.

Cárdenas, M., Meza, P., Lagues, K. \& Yañez, S. (2010). Adaptación y validación de la escala de Orientación a la Dominancia Social (SDO) en una muestra chilena. Universitas Psychologica, 9(1), 161-168. https://doi.org/10.11144/Javeriana.upsy9-1.aveo

Carretero-Dios, H. \& Pérez, C. (2007). Normas para el desarrollo y revisión de estudios instrumentales: consideraciones sobre la selección de tests en la investigación psicológica. International Journal of Clinical and Health Psychology, 7(3), 863-882. https://aepc.es/ijchp/NDREI07_es.pdf

Carvacho, H., Manzi, J., Haye, A., González, R. \& Cornejo, M. (2013). Consenso y disenso en la memoria histórica y en las actitudes hacia la reparación en tres generaciones de chilenos. Psykhe, 22(2), 33-47. https://doi.org/10.7764/psykhe.22.2.601

Cheung, G. W. \& Rensvold, R. B. (2002). Evaluating goodness-of-fit indexes for testing measurement invariance. Structural Equation Modeling, 9(2), 233-255. https://doi.org/10.1207/S15328007SEM0902_5

Cotterill, S., Sidanius, J., Bhardwaj, A. \& Kumar, V. (2014). Ideological support for the Indian caste system: Social dominance orientation, right-wing authoritarianism and karma. Journal of Social and Political Psychology, 2(1), 98-116. https://doi.org/10.5964/jspp.v2i1.171

Cronbach, L. J. (1995). Giving method variance its due. En P. E. Shrout \& S. T. Fiske (Eds.), Personality research, methods, and theory: A festschrift honoring Donald W. Fiske (pp. 145-157). Lawrence Erlbaum. https://doi.org/10.4324/9781315806815

Duckitt, J. (2006). Differential effects of right wing authoritarianism and social dominance orientation on outgroup attitudes and their mediation by threat from and competitiveness to outgroups. Personality and Social Psychology Bulletin, 32(5), 684-696. https://doi.org/10.1177/0146167205284282

Duckitt, J. \& Sibley, C. G. (2007). Right wing authoritarianism, social dominance orientation and the dimensions of generalized prejudice. European Journal of Personality, 21(2), 113-130. https://doi.org/10.1002/per.614

Ellenbroek, M., Verkuyten, M., Thijs, J. \& Poppe, E. (2014). The fairness of national decision-making procedures: The views of adolescents in 18 European countries. Journal of Community \& Applied Social Psychology, 24(6), 503-517. https://doi.org/10.1002/casp.2189

Etchezahar, E., Prado-Gascó, V., Jaume, L. \& Brussino, S. (2014). Validación argentina de la escala de Orientación a la Dominancia Social. Revista Latinoamericana de Psicología, 46(1), 35-43. https://doi.org/10.1016/S0120-0534(14)70004-4

Friesen, J. P., Laurin, K., Shepherd, S., Gaucher, D. \& Kay, A. C. (2019). System justification: Experimental evidence, its contextual nature, and implications for social change. British Journal of Social Psychology, 58(2), 315-339. https://doi.org/10.1111/bjso.12278

García-Sánchez, E., Castillo, J., Willis, G. B. \& Rodríguez-Bailón, R. (2021). The two faces of support for redistribution in Colombia: Taxing the wealthy or assisting people in poverty [Manuscrito enviado para publicación]. Departamento de Psicología Social, Universidad de Granada.

García-Sánchez, E., Willis, G. B., Rodríguez-Bailón, R., Palacio Sañudo, J., Polo, J. D. \& Rentería Pérez, E. (2018). Perceptions of economic inequality and support for redistribution: The role of existential and utopian standards. Social Justice Research, 31(4), 335-354. https://doi.org/10.1007/s11211-018-0317-6

Grina, J., Bergh, R., Akrami, N. \& Sidanius, J. (2016). Political orientation and dominance: Are people on the political right more dominant? Personality and Individual Differences, 94, 113-117. https://doi.org/10.1016/i.paid.2016.01.015

Hindriks, P., Verkuyten, M. \& Coenders, M. (2014). Dimensions of social dominance orientation: The roles of legitimizing myths and national identification. European Journal of Personality, 28(6), 538-549. https://doi.org/10.1002/per.1955 
Hirschfeld, G. \& von Brachel, R. (2014). Improving multiple-group confirmatory factor analysis in R - A tutorial in measurement invariance with continuous and ordinal indicators. Practical Assessment, Research and Evaluation, 19 , Artículo 7. https://doi.org/10.7275/qazy-2946

Ho, A. K., Sidanius, J., Kteily, N., Sheehy-Skeffington, J., Pratto, F., Henkel, K. E., Foels, R. \& Stewart, A. L. (2015). The nature of social dominance orientation: Theorizing and measuring preferences for intergroup inequality using the new $\mathrm{SDO}_{7} \mathrm{scale}$. Journal of Personality and Social Psychology, 109(6), 1003-1028. https://doi.org/10.1037/pspi0000033

Janos, E., Espinosa, A. \& Pacheco, M. (2018). Bases ideológicas de la percepción del sistema normativo y el cinismo político en adultos de sectores urbanos del Perú. Psykhe, 27(1), Artículo 2. https://doi.org/10.7764/psykhe.27.1.1176

Jaume, L. C., Etchezahar, E. \& Cervone, N. (2012). La justificación del sistema económico y su relación con la orientación a la dominancia social. Boletín de Psicología de España, 106, 81-91. https://www.uv.es/seoane/boletin/previos/N106-5.pdf

Jost, J. T. (2019). A quarter century of system justification theory: Questions, answers, criticisms, and societal applications. British Journal of Social Psychology, 58(2), 263-314. https://doi.org/10.1111/bjso.12297

Jost, J. T., Blount, S., Pfeffer, J. \& Hunyady, G. (2003). Fair market ideology: Its cognitive-motivational underpinnings. Research in Organizational Behavior, 25, 53-91. https://doi.org/10.1016/S0191-3085(03)25002-4

Jost, J. T. \& Thompson, E. P. (2000). Group-based dominance and opposition to equality as independent predictors of self-esteem, ethnocentrism, and social policy attitudes among African Americans and European Americans. Journal of Experimental Social Psychology, 36(3), 209-232. https://doi.org/10.1006/jesp.1999.1403

Kline, R. B. (2016). Principles and practice of structural equation modeling: Fourth edition. Guilford Press.

Kteily, N., Bruneau, E., Waytz, A. \& Cotterill, S. (2015). The ascent of man: Theoretical and empirical evidence for blatant dehumanization. Journal of Personality and Social Psychology, 109(5), 901-931. https://doi.org/10.1037/pspp0000048

Kunst, J. R., Fischer, R., Sidanius, J. \& Thomsen, L. (2017). Preferences for group dominance track and mediate the effects of macrolevel social inequality and violence across societies. Proceedings of the National Academy of Sciences of the United States of America, 114(21), 5407-5412. https://doi.org/10.1073/pnas.1616572114

Larsson, M. R., Björklund, F. \& Bäckström, M. (2012). Right-wing authoritarianism is a risk factor of torture-like abuse, but so is social dominance orientation. Personality and Individual Differences, 53(7), 927-929. https://doi.org/10.1016/j.paid.2012.06.015

Martinovic, B. \& Verkuyten, M. (2013). 'We were here first, so we determine the rules of the game': Autochthony and prejudice towards out-groups. European Journal of Social Psychology, 43(7), 637-647. https://doi.org/10.1002/ejsp.1980

Muñiz, J., Elosua, P. \& Hambleton, R. K. (2013). Directrices para la traducción y adaptación de los tests: segunda edición. Psicothema, 25(2), 151-157. https://doi.org/10.7334/psicothema2013.24

Pratto, F., Çidam, A., Stewart, A. L., Bou Zeineddine, F., Aranda, M., Aiello, A., Chryssochoou, X., Cichocka, A., Cohrs, J. C., Durrheim, K., Eicher, V., Foels, R., Górska, P., Lee, I. -C., Licata, L., Liu, J. H., Li, L., Meyer, I., Morselli, D. ... Henkel, K. E. (2013). Social dominance in context and in individuals: Contextual moderation of robust effects of social dominance orientation in 15 languages and 20 countries. Social Psychological and Personality Science, 4(5), 587-599. https://doi.org/10.1177/1948550612473663

Pratto, F., Sidanius, J., Stallworth, L. M. \& Malle, B. F. (1994). Social dominance orientation: A personality variable predicting social and political attitudes. Journal of Personality and Social Psychology, 67(4), 741-763. https://doi.org/10.1037/0022-3514.67.4.741

R Development Core Team. (2019). R: A language and environment for statistical computing. R Foundation for Statistical Computing. https://www.R-project.org

Revelle, W. (2018). psych: Procedures for psychological, psychometric, and personality research (Versión 2.0). Northwestern University. https://personality-project.org/r/psych/

Rosseel, Y. (2012). lavaan: An R package for structural equation modeling. Journal of Statistical Software, 48, Artículo 2. https://doi.org/10.18637/jss.v048.i02

Sibley, C. G. \& Duckitt, J. (2010). The ideological legitimation of the status quo: Longitudinal tests of a social dominance model. Political Psychology, 31(1), 109-137. https://doi.org/10.1111/j.1467-9221.2009.00747.x

Sidanius, J., Cotterill, S., Sheehy-Skeffington, J., Kteily, N. \& Carvacho, H. (2017). Social dominance theory: Explorations in the psychology of oppression. En C. G. Sibley \& F. K. Barlow (Eds.), The Cambridge Handbook of the Psychology of Prejudice (pp. 149-187). Cambridge University Press. https://doi.org/10.1017/9781316161579.008

Sidanius, J., Levin, S., Liu, J. \& Pratto, F. (2000). Social dominance orientation, anti-egalitarianism and the political psychology of gender: An extension and cross-cultural replication. European Journal of Social Psychology, 30(1), 41-67. https://doi.org/10.1002/(SICI)1099-0992(200001/02)30:1<41::AID-EJSP976>3.0.CO;2-O

Sidanius, J. \& Pratto, F. (1999). Social Dominance: An intergroup theory of social hierarchy and oppression. Cambridge University Press. https://doi.org/10.1017/CBO9781139175043

Silván Ferrero, M. P. \& Bustillos, A. (2007). Adaptación de la escala de Orientación a la Dominancia Social al castellano: validación de la dominancia grupal y la oposición a la igualdad como factores subyacentes. Revista de Psicología Social, 22(1), 316. https://doi.org/10.1174/021347407779697485

Sirlopú, D., Melipillán, R., Sánchez, A. \& Valdés, C. (2015). ¿Malos para aceptar la diversidad? Predictores socio-demográficos y psicológicos de las actitudes hacia el multiculturalismo en Chile. Psykhe, 24(2), Artículo 6. https://doi.org/10.7764/psykhe.24.2.714

Ungaretti, J. \& Etchezahar, E. (2016). Visiones del mundo, autoritarismo y dominancia en diferentes expresiones de prejuicio. Acta de Investigación Psicológica, 6(3), 2500-2508. https://doi.org/10.1016/j.aipprr.2016.11.004

Vargas-Salfate, S., Paez, D., Liu, J. H., Pratto, F. \& Gil de Zúñiga, H. (2018). A comparison of social dominance theory and system justification: The role of social status in 19 nations. Personality and Social Psychology Bulletin, 44(7), $1060-1076$. https://doi.org/10.1177/0146167218757455 


\section{Anexo}

Tabla A

Traducción de la Escala de Orientación a la Dominancia Social 7 al Castellano

\begin{tabular}{|c|c|}
\hline \multicolumn{2}{|c|}{ Dominancia } \\
\hline Item en la versión original en inglés & Ítem en la versión adaptada al castellano \\
\hline Some groups of people must be kept in their place. & $\begin{array}{l}\text { Algunos grupos de personas deberían ser mantenidos en } \\
\text { su posición social. }\end{array}$ \\
\hline $\begin{array}{l}\text { It's probably a good thing that certain groups are at the } \\
\text { top and other groups are at the bottom. }\end{array}$ & $\begin{array}{l}\text { Probablemente sea bueno que en la sociedad ciertos } \\
\text { grupos de personas están arriba y otros grupos de } \\
\text { personas están abajo. }\end{array}$ \\
\hline $\begin{array}{l}\text { An ideal society requires some groups to be on top and } \\
\text { others to be on the bottom. }\end{array}$ & $\begin{array}{l}\text { Una sociedad ideal requiere que algunos grupos de } \\
\text { personas están arriba y otros están abajo. }\end{array}$ \\
\hline $\begin{array}{l}\text { Some groups of people are simply inferior to other } \\
\text { groups. }\end{array}$ & $\begin{array}{l}\text { Algunos grupos de personas son simplemente inferiores } \\
\text { a otros grupos de personas. }\end{array}$ \\
\hline $\begin{array}{l}\text { Groups at the bottom are just as deserving as groups at } \\
\text { the top. (r) }\end{array}$ & $\begin{array}{l}\text { "Los grupos de personas en posiciones inferiores son tan } \\
\text { merecedores como los grupos de personas en posiciones } \\
\text { superiores. }(\mathrm{r})\end{array}$ \\
\hline No one group should dominate in society. (r) & $\begin{array}{l}\text { Ningún grupo de personas debería dominar en la } \\
\text { sociedad. (r) }\end{array}$ \\
\hline $\begin{array}{l}\text { Groups at the bottom should not have to stay in their } \\
\text { place. (r) }\end{array}$ & $\begin{array}{l}{ }^{*} \text { Los grupos de personas en posiciones inferiores no } \\
\text { tienen que quedarse en su posición. }(\mathrm{r})\end{array}$ \\
\hline Group dominance is a poor principle. (r) & $\begin{array}{l}\text { La dominación de unos grupos de personas sobre otros } \\
\text { grupos es un principio mediocre. }(\mathrm{r})\end{array}$ \\
\hline
\end{tabular}

Anti-igualitarismo

\begin{tabular}{|c|c|}
\hline Item en la versión original en inglés & Ítem en la versión adaptada al castellano \\
\hline We should not push for group equality. & $\begin{array}{l}\text { No deberíamos intentar garantizar que cada grupo de } \\
\text { personas tenga la misma calidad de vida. }\end{array}$ \\
\hline $\begin{array}{l}\text { We shouldn't try to guarantee that every group has the } \\
\text { same quality of life. }\end{array}$ & $\begin{array}{l}\text { No deberíamos promover la igualdad entre grupos de } \\
\text { personas. }\end{array}$ \\
\hline It is unjust to try to make groups equal. & Es injusto intentar igualar a los grupos de personas. \\
\hline Group equality should not be our primary goal. & $\begin{array}{l}\text { La igualdad entre grupos de personas no debería ser } \\
\text { nuestro objetivo principal. }\end{array}$ \\
\hline $\begin{array}{l}\text { We should work to give all groups an equal chance to } \\
\text { succeed. (r) }\end{array}$ & $\begin{array}{l}\text { *Deberíamos trabajar para dar a todos los grupos de } \\
\text { personas igualdad de oportunidades para tener éxito. } \\
\text { (r) }\end{array}$ \\
\hline $\begin{array}{l}\text { We should do what we can to equalize conditions for } \\
\text { different groups. (r) }\end{array}$ & $\begin{array}{l}\text { Deberíamos hacer todo lo posible para igualar las } \\
\text { condiciones de los diferentes grupos de personas. (r) }\end{array}$ \\
\hline $\begin{array}{l}\text { No matter how much effort it takes, we ought to strive to } \\
\text { ensure that all groups have the same chance in life. (r) }\end{array}$ & $\begin{array}{l}\text { *No importa cuánto esfuerzo requiera, debemos luchar } \\
\text { para garantizar que todos los grupos de personas } \\
\text { tengan las mismas oportunidades en la vida. (r) }\end{array}$ \\
\hline Group equality should be our ideal. (r) & $\begin{array}{l}\text { La igualdad entre grupos de personas debería ser } \\
\text { nuestro ideal. (r) }\end{array}$ \\
\hline
\end{tabular}

Nota. $r$ = ítems en negativo que deben ser revertidos; 'ítems eliminados de la escala para el contexto colombiano. 
Tabla B

Variables e Ítems Incluidos en los Estudio 1 y 2 para Evaluar la Validez Predictiva de la $\mathrm{SDO}_{7}$

\begin{tabular}{|c|c|c|}
\hline Variable & Estudio & Ítem \\
\hline \multirow{6}{*}{$\begin{array}{l}\text { Justificación del sistema } \\
\text { económico }\end{array}$} & $1 \mathrm{y} 2$ & Si la gente trabaja duro, casi siempre consigue lo que quiere. \\
\hline & 1 & $\begin{array}{l}\text { La mayoría de la gente que no progresa en nuestra sociedad no debería culpar al sistema: } \\
\text { ellos son los únicos culpables. }\end{array}$ \\
\hline & 1 у 2 & La posición económica de las personas es un producto de sus logros. \\
\hline & 1 & Si alguien se esfuerza lo suficiente, puede ascender en la escala social. \\
\hline & 1 & Hay mucha gente que es pobre porque no le gusta trabajar. \\
\hline & 2 & La brecha entre clases sociales refleja las diferencias en el orden natural de las cosas. \\
\hline \multirow[t]{2}{*}{ Patriotismo } & 2 & Siento un gran orgullo por nuestro país Colombia. \\
\hline & 2 & $\begin{array}{l}\text { Aunque a veces no esté de acuerdo con el Gobierno, mi compromiso con Colombia siempre } \\
\text { es muy fuerte. }\end{array}$ \\
\hline $\begin{array}{l}\text { Ideología política (izquierda- } \\
\text { derecha) }\end{array}$ & 1 y 2 & $\begin{array}{l}\text { Cuando se habla de política generalmente se usan categorías como "izquierda" y } \\
\text { "derecha". Según estas categorías, escriba una X encima de la casilla con la que usted } \\
\text { mejor se identificaría. }\end{array}$ \\
\hline Apoyo a la protesta social & 1 y 2 & $\begin{array}{l}\text { Hacer marchas de protesta y manifestaciones públicas apoyando los derechos de los } \\
\text { grupos más desfavorecidos en Colombia. }\end{array}$ \\
\hline $\begin{array}{l}\text { Apoyo a la solución militar } \\
\text { del conflicto armado }\end{array}$ & 1 y 2 & La solución militar es la mejor forma de resolver el conflicto armado en Colombia. \\
\hline $\begin{array}{l}\text { Preocupación por la } \\
\text { desigualdad }\end{array}$ & 1 y 2 & Las diferencias de ingresos económicos en Colombia son demasiado grandes. \\
\hline $\begin{array}{l}\text { Apoyo al matrimonio } \\
\text { igualitario (homosexual) }\end{array}$ & 1 & Legalizar el matrimonio entre personas del mismo sexo. \\
\hline $\begin{array}{l}\text { Apoyo al castigo físico a } \\
\text { los/as niños/as }\end{array}$ & 1 & $\begin{array}{l}\text { Está bien que los padres puedan castigar físicamente a sus hijos como una forma de } \\
\text { disciplinarlos. }\end{array}$ \\
\hline $\begin{array}{l}\text { Justificación de la } \\
\text { desigualdad }\end{array}$ & 1 & $\begin{array}{l}\text { Si los ingresos económicos fueran más igualitarios, las personas estarían menos } \\
\text { motivadas a trabajar duro. }\end{array}$ \\
\hline Apoyo a la inversión social & 1 & $\begin{array}{l}\text { El Gobierno debería incrementar el gasto público en subsidios para las personas más } \\
\text { desfavorecidas. }\end{array}$ \\
\hline $\begin{array}{l}\text { Apoyo a la inclusión } \\
\text { educativa }\end{array}$ & 1 & $\begin{array}{l}\text { Reservar cupos en las universidades para personas provenientes de comunidades } \\
\text { vulnerables. }\end{array}$ \\
\hline $\begin{array}{l}\text { Apoyo a la redistribución } \\
\text { (Medida 1) }\end{array}$ & 1 & $\begin{array}{l}\text { El Gobierno tiene la responsabilidad de reducir las diferencias de ingresos económicos } \\
\text { entre quienes tienen más y los que tienen menos. }\end{array}$ \\
\hline \multirow[t]{7}{*}{$\begin{array}{l}\text { Actitudes hacia la } \\
\text { redistribución (Medida 2) }\end{array}$} & 2 & $\begin{array}{l}\text { El Gobierno tiene la responsabilidad de reducir las diferencias de ingresos entre los que } \\
\text { tienen más y los que tienen menos. }\end{array}$ \\
\hline & 2 & El Gobierno debería gastar más dinero en subsidios para los pobres. \\
\hline & 2 & $\begin{array}{l}\text { El Gobierno debería imponer mayores impuestos a las personas con más ingresos } \\
\text { económicos. }\end{array}$ \\
\hline & 2 & Se deberían reservar cupos en universidades para las personas más desfavorecidas. \\
\hline & 2 & $\begin{array}{l}\text { Existe una gran necesidad de redistribuir la riqueza de aquellos que tienen más, hacia } \\
\text { aquellos que tienen menos. }\end{array}$ \\
\hline & 2 & $\begin{array}{l}\text { No hay ninguna necesidad de cambiar la distribución de ingresos económicos en Colombia. } \\
\text { (recodificada) }\end{array}$ \\
\hline & 2 & Las personas con más riqueza deberían ayudar más a las personas más necesitadas. \\
\hline Apoyo a la pena de muerte & 1 & Implementar la pena capital (pena de muerte) en Colombia. \\
\hline Apoyo a penas más fuertes & 2 & En Colombia deberían emplearse castigos mucho más duros contra los criminales. \\
\hline Valores: Disciplina & 1 & Autodisciplina (autocontrol, resistirse a la tentación). \\
\hline Valores: Honrar padres & 1 & Honrar a los padres y mayores (mostrar mucho respeto). \\
\hline Valores: Igualdad & 1 & Igualdad (igualdad de oportunidades para todos). \\
\hline Valores: Paz & 1 & Un mundo en paz (libre de guerras y conflictos). \\
\hline Valores: Justicia & 1 & La justicia social (corregir las injusticias y cuidar a los vulnerables). \\
\hline Valores: Autoridad & 1 & Autoridad (el derecho de algunos para liderar o dirigir). \\
\hline Valores: Impacto & 1 & Influencia (tener un impacto sobre las personas y eventos). \\
\hline Valores: Riqueza & 1 & Riqueza (posesiones materiales, dinero). \\
\hline $\begin{array}{l}\text { Estatus objetivo por } \\
\text { ingresos familiares }\end{array}$ & 1 y 2 & $\begin{array}{l}\text { Señale la cantidad aproximada de ingresos mensuales netos que tiene en su núcleo familiar. } \\
\text { Tenga en cuenta todas las fuentes de ingresos (sueldos, pensiones, becas, rentas de } \\
\text { alquileres, etc.) de las personas que aportan al sostenimiento de la vivienda eF8.2 }\end{array}$ \\
\hline Estatus subjetivo & 1 y 2 & Status subjetivo. \\
\hline Estatus por nivel educativo & 2 & $\begin{array}{l}\text { Por favor, señale el último nivel de estudios que ha alcanzado o que está cursando } \\
\text { actualmente. }\end{array}$ \\
\hline
\end{tabular}


Tabla C

Tabla de Correlaciones de Pearson entre los Ítems de la Escala SDO

\begin{tabular}{|c|c|c|c|c|c|c|c|c|c|c|c|c|c|c|c|c|}
\hline & $\operatorname{dom} 1$ & $\operatorname{dom} 2$ & $\operatorname{dom} 3$ & $\operatorname{dom} 4$ & $\operatorname{dom} 5$ & dom6 & $\operatorname{dom} 7$ & $\operatorname{dom} 8$ & antieg1 & antieg2 & antieg3 & antieg4 & antieg5 & antieg6 & antieg7 & antieg8 \\
\hline $\operatorname{dom} 1$ & & $0,266^{* * *}$ & $730^{* * *}$ & $0,304^{* * *}$ & $382^{* * *}$ & $0,119^{* * *}$ & $0,447^{* * *}$ & $176^{* * *}$ & $0,318^{* * *}$ & $0,365^{* * *}$ & $0,164^{* * *}$ & $0,385^{* * *}$ & $0,235^{* * *}$ & $0,332^{* * *}$ &, $242^{* * *}$ & $0,398^{* * *}$ \\
\hline $\operatorname{dom} 2$ & $0,247^{* * *}$ & & $0,308^{* * *}$ & $0,126^{* * *}$ & $0,195^{* * *}$ & $0,122^{* * *}$ & $0,303^{* * *}$ & 0,032 & $0,158^{* * *}$ & $0,133^{* * *}$ & $0,155^{\text {*** }}$ & $0,181^{* * *}$ & $0,178^{* * *}$ & $0,175^{* * *}$ & $0,112^{* * *}$ & $0,177^{* * *}$ \\
\hline $\operatorname{dom} 3$ & $0,673^{* * *}$ & $0,298^{* * *}$ & & $0,356^{\text {*** }}$ & $0,421^{* * *}$ & $0,107^{* *}$ & $0,410^{* * *}$ & $0,139^{* * *}$ & $0,354^{* * *}$ & $0,379^{* * *}$ & $0,136^{\text {*** }}$ & $0,391^{* * *}$ & $0,227^{\text {***}}$ & $0,343^{* * *}$ & $0,264^{* * *}$ & $0,375^{* * *}$ \\
\hline $\operatorname{dom} 4$ & $0,179^{* * *}$ & $0,180^{* * *}$ & $0,254^{* * *}$ & & $0,275^{* * *}$ & $0,130^{* * *}$ & $0,247^{* * *}$ & $0,100^{* *}$ & $0,169^{* * *}$ & $0,219^{* * *}$ & $0,177^{* * *}$ & $0,239^{* * *}$ & $0,161^{* * *}$ & $0,244^{* * *}$ & $0,162^{* * *}$ & $0,187^{* * *}$ \\
\hline $\operatorname{dom} 5$ & $0,318^{* * *}$ & $0,156^{* *}$ & $0,339^{* * *}$ & $0,238^{* * *}$ & & $0,194^{* * *}$ & $0,284^{* * *}$ & $0,189^{* * *}$ & $0,254^{* * *}$ & $0,259^{* * *}$ & $0,251^{* * *}$ & $0,293^{* * *}$ & $0,245^{* * *}$ & $0,344^{* * *}$ & $0,266^{* * *}$ & $0,329^{* * *}$ \\
\hline dom 6 & 0,086 & 0,019 & $0,118^{*}$ & 0,062 & $0,116^{*}$ & & $0,257^{* * *}$ & $0,154^{* * *}$ & $0,197^{* * *}$ & $0,093^{* *}$ & $0,224^{* * *}$ & $0,187^{* * *}$ & $0,259^{* * *}$ & $0,245^{* * *}$ & $0,209^{* * *}$ & $0,277^{* * *}$ \\
\hline $\operatorname{dom} 7$ & $0,367^{\text {*** }}$ & $0,406^{\text {*** }}$ & $0,495^{* * *}$ & $0,154^{* *}$ & $0,294^{* * *}$ & $0,143^{* *}$ & & $0,178^{* * *}$ & $0,354^{* * *}$ & $0,222^{* * *}$ & $0,249^{* * *}$ & $0,264^{* * *}$ & $0,284^{* * *}$ & $0,315^{* * *}$ & $0,247^{* * *}$ & $0,421^{* * *}$ \\
\hline $\operatorname{dom} 8$ & $0,265^{* * *}$ & $0,136^{* *}$ & $0,189^{* * *}$ & 0,053 & $0,217^{* * *}$ & $0,201^{* * *}$ & $0,156^{* *}$ & & $0,180^{* * *}$ & $0,164^{* * *}$ & $0,146^{* * *}$ & $0,193^{* * *}$ & $0,246^{* * *}$ & $0,199^{* * *}$ & $0,139^{* * *}$ & $0,328^{* * *}$ \\
\hline anti-ig1 & $0,391^{* * *}$ & $0,221^{* * *}$ & $0,364^{* * *}$ & 0,090 & $0,207^{* * *}$ & $0,123^{*}$ & $0,265^{* * *}$ & $0,276^{* * *}$ & & $0,356^{* * *}$ & $0,274^{* * *}$ & $0,454^{* * *}$ & $0,267^{\text {*** }}$ & $0,490^{* * *}$ & $0,337^{* * *}$ & $0,505^{* * *}$ \\
\hline anti-ig2 & $0,252^{* * *}$ & 0,082 & $0,283^{* * *}$ & $0,147^{\text {** }}$ & $0,263^{* * *}$ & $0,136^{* *}$ & $0,115^{*}$ & $0,241^{* * *}$ & $0,395^{* * *}$ & & $0,090^{* *}$ & $0,390^{* * *}$ & $0,169^{* * *}$ & $0,367^{* * *}$ & $0,254^{* * *}$ & $0,323^{* * *}$ \\
\hline anti-ig3 & $0,249^{* * *}$ & $0,129^{*}$ & $0,181^{* * *}$ & 0,061 & $0,153^{* *}$ & $0,155^{* *}$ & 0,100 & $0,227^{* * *}$ & $0,320^{* * *}$ & $0,206^{* * *}$ & & $0,205^{* * *}$ & $0,505^{* * *}$ & $0,285^{* * *}$ & $0,299^{* * *}$ & $0,362^{* * *}$ \\
\hline anti-ig4 & $0,330^{* * *}$ & $0,163^{* *}$ & $0,357^{* * *}$ & $0,206^{* * *}$ & $0,337^{* * *}$ & 0,083 & $0,242^{* * *}$ & $0,271^{* * *}$ & $0,432^{* * *}$ & $0,389^{* * *}$ & $0,297^{* * *}$ & & $0,271^{* * *}$ & $0,475^{* * *}$ & $0,332^{* * *}$ & $0,431^{* * *}$ \\
\hline anti-ig5 & $0,297^{\text {***}}$ & $0,128^{*}$ & $50^{* * *}$ & 0,072 & 0,266 & $0,193^{* * *}$ & 0,20 & $0,20 \leqq$ & $0,34 \mathrm{c}$ & 0,1 & 0,6 & $0,297^{* * *}$ & & $0,321^{* * *}$ & 0,3 & 0,4 \\
\hline anti-ig6 & $0,392^{* * *}$ & $0,119^{*}$ & $0,341^{* * *}$ & $0,115^{*}$ & $0,258^{* * *}$ & $0,149^{* *}$ & $0,208^{* * *}$ & $0,224^{* * *}$ & $0,446^{* * *}$ & $0,273^{* * *}$ & $0,296^{* * *}$ & $0,412^{* * *}$ & $0,288^{* * *}$ & & $0,483^{* * *}$ & $0,488^{* * *}$ \\
\hline anti-ig7 & $0,287^{* * *}$ & $0,139^{* *}$ & $0,299^{* * *}$ & $0,115^{*}$ & $0,323^{* * *}$ & $0,113^{*}$ & $0,202^{* * *}$ & $0,170^{* *}$ & $0,368^{* * *}$ & $0,313^{* * *}$ & $0,280^{* * *}$ & $0,415^{* * *}$ & $0,283^{* * *}$ & $0,528^{* * *}$ & & $0,429^{* * *}$ \\
\hline anti-ig8 & $0,430^{* * *}$ & $0,199^{* * *}$ & $0,407^{* * *}$ & $0,109^{*}$ & $0,335^{* * *}$ & 0,101 & $0,355^{* * *}$ & $0,405^{* * *}$ & $0,583^{* * *}$ & $0,340^{* * *}$ & $0,387^{* * * *}$ & $0,438^{* * *}$ & $0,477^{\text {***}}$ & $0,477^{* * *}$ & $0,435^{* * *}$ & \\
\hline
\end{tabular}

Nota. *** $p<0,001 ; * * p<0,01 ; * p<0,05$ 
Fecha de recepción: Octubre de 2019.

Fecha de aceptación: Mayo de 2021. 